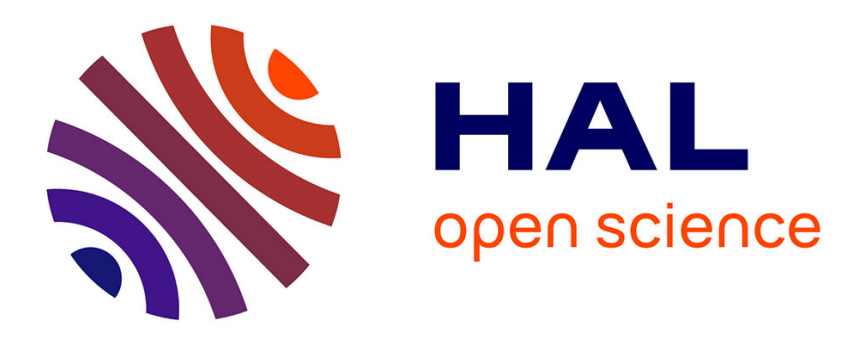

\title{
Médaillons d'applique à sujets religieux des vallées du Rhône et de l'Allier
}

\author{
Amable Audin, Hugues Vertet
}

\section{To cite this version:}

Amable Audin, Hugues Vertet. Médaillons d'applique à sujets religieux des vallées du Rhône et de l'Allier. Gallia - Fouilles et monuments archéologiques en France métropolitaine, 1972, 30 (2), pp.235258. 10.3406/galia.1972.2602 . hal-01934896

\section{HAL Id: hal-01934896 https://hal.science/hal-01934896}

Submitted on 11 Mar 2020

HAL is a multi-disciplinary open access archive for the deposit and dissemination of scientific research documents, whether they are published or not. The documents may come from teaching and research institutions in France or abroad, or from public or private research centers.
L'archive ouverte pluridisciplinaire HAL, est destinée au dépôt et à la diffusion de documents scientifiques de niveau recherche, publiés ou non, émanant des établissements d'enseignement et de recherche français ou étrangers, des laboratoires publics ou privés.

\section{(ㅇ)(1) $\$$}

Distributed under a Creative Commons Attribution - NonCommercial - NoDerivatives| 4.0 


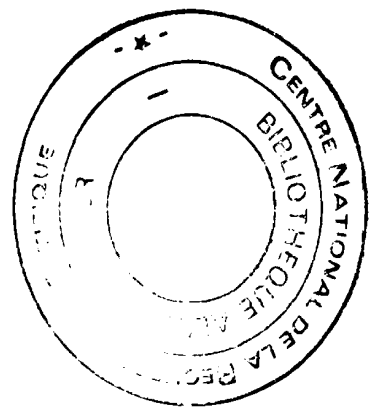

\title{
MÉDAILLONS D'APPLIQUE À SUJETS RELIGIEUX DES VALLÉES DU RHÔNE ET DE L'ALLIER
}

\author{
par Amable AUDIN ef Hugues VERTET
}

On sait depuis longtemps que les médaillons d'applique rhodaniens présentent une grande variété de scènes ayant trait aux religions orientales comme à la mythologie classiquel. La découverte récente de surmoulages de sènes isiaques dans les ateliers arvernes a attiré l'attention sur la diffusion inattendue de thèmes égyptiens dans le Centre de la Gaule². Des fouilles faites dans les ateliers de Lezoux, aussi bien que l'étude des réserves des Musées de Vichy et de Moulins, permettent d'ajouter d'autres documents à ceux que nous connaissions déjà et de percevoir à quel point la comparaison des productions lyonnaises et des productions arvernes ouvre la voie à des recherches nouvelles.

\section{I. - Le médaillox de: supplice de llarsyas}

Ce médaillon a pu être restitué à partir d'une douzaine de fragments éparpillés en France. On soulignera en premier licu sa valeur artistique. Et, puisqu'il s'agit d'une œurre spécifiquement gallo-romaine, elle témoigne d'un sens esthétique très sûr, moins par l'heureuse composition du sujet qui pourrait avoir été inspiré par un morlèle étranger, que par la qualité de l'exécution, singulièrement adroite dans le personnage de l'esclave scythe, et noble dans celui d'Apollon. En second lieu, cette œuvre manifeste une connaissance approfondie de la mythologie classique, d'abord chez l'auteur du médaillon, mais aussi dans sa clientèle, car il n'aurait pas pris le soin de modeler un sujet de cette importance et de cette grandeur s'il n'avait été certain de rencontrer l'approbation de son public, et l'on verra qu'elle fut large.

1 J. Déchelette, Les vases céramiques ornés de la Caule romaine, 1964 (II, 4 " partie, vase's à reliefs d'applique, série $B$, vases de la vallée du Rhòne, p. $235-307$ et supplément, p. 345-352 et pl. 11 , no 77 , et pl. IV, vase Sallier ; P. Wuntrivine et A. Aunis, Médaillons d'applique gallo-romains de la vallee du Rhìne, 1952.

2 H. Veнтет, Observations sur les vases a médaillons d'applique de la vallée du Rhine, dans (iallia, XXVII, 1969 , p. 124-126. 
Pour nous, il n'y a pas de doute qu'il appartienne à l'école lyonnaise, et, plus précisément, qu'il soit l'œuvre de ce Felix qui en fut le plus prestigieux artisan, tant par la valeur plastique de ses productions que par l'ouverture qu'il avait sur les mythes de l'Olympe ${ }^{3}$. Fn outre, un certain nombre de détails permettent d'étayer plus fermement cette attribution. En revanche, la dimension du médaillon (140 mm de diamètre) en fait, sinon un cas unique dans l'œuvre de Felix, du moins un cas exceptionnel. Ses productions oscillent entre 60 et $80 \mathrm{~mm}$, mais on se souviendra que le médaillon de Cavillargues, qui lui revient sûrement, mesure $167 \mathrm{~mm}$ de diamètre.

Enfin, l'exploitation de l'original lyonnais par les ateliers arvernes est remarquable. Exploitation intense, si l'on considère le nombre des fragments trouvés à Lezoux. Cettr observation débouche sur des considérations techniques : en effet, le surmoulage d'un médaillon entraìne le retrait au séchage des nouvelles épreuves, et si, par exemple, nous mesurons sur l'original lyonnais la distance qui va du sommet de l'épaule du Scythe à la bande du sol, $30 \mathrm{~mm}$, on constate que, sur un surmoulage de Lezoux, elle ne dépasse plus $26 \mathrm{~mm}$, ce qui implique un retrait de $15 \%$ qui, d'ailleurs, provient peut-être de plusieurs surmoulages additionnés. Cela joue même pour des médaillons réalisés à Leezoux : sur le beau fragment d'Apollon, la cithare et son support mesurent $39 \mathrm{~mm}$ de haut, et 35 seulement sur un autre.

Pour autant qu'on le puisse apprécier, ce médaillon original mesurait $140 \mathrm{~mm}$ de diamètre (fig. 1). Son carlre, comme tous ceux de Felix, était constitué par un simple tore. Le médaillon était coupé par une bande horizontale qui laissait en exergue le quart inférièr. Cette bande, qui sert de sol à la scène, est bordée de deux moulures, celle du haut ctant piqueté de points (fig. 2). La partie centrale est un treillis de filets, serrés lorsqu'ils sont inclinés en arrière, plus espacés dans le sens inverse, détails qui n'apparaissent que sur lo fragment de I,yon.

Longeant le cadre en sa partie gauche depuis le sol presque jusqu'au sommet du médaillon, se voit le trone noueux d'un pin, au sommet duquel Marsyas est attaché par les poignets. Entre l'arbre et lui, un personnage entièrement vêtu hisse au moyen d'une corde le malheureux silène barbu dont les pieds ont déjà quitté le sol et dont le corps nu s'étire en longueur. En dessous du tireur de corde, un personnage nu est accroupi. Regardant la victime, il a de longs chereux hirsutes. Son corps porte sur le genou droit, le galuche étant relevé, et de ses deux mains, il aiguise sur une meule le couteau destiné à écorcher vif l'adversaire d'Apollon. Placée verticalement entre le pin et lui, la légende SCYYTHES le désigne comme l'esclave scythe chargé de l'affreuse besogne. Au centre du médaillon, un quatrième individu, de petite taille, vêtu d'une scule chlamỵde qui flotte sur ses épaules, se précipite vers la droite pour se jeter aux pieds d'Apollon et demander la grâce du silène. La légende verticale placée devant lui, OLIIIPTS, le désigne comme le disciple et ami de Marsyas.

'Toute la partie droite du médaillon est occupée par l'image d'Apollon. I'une taille

3 A. Audr: et W. Binsfreln, Médaillons dapplique rhodaniens du Musée de Cologne, dans Kölner Jahrbuch, 1964, p. 14-17, p. 14. 


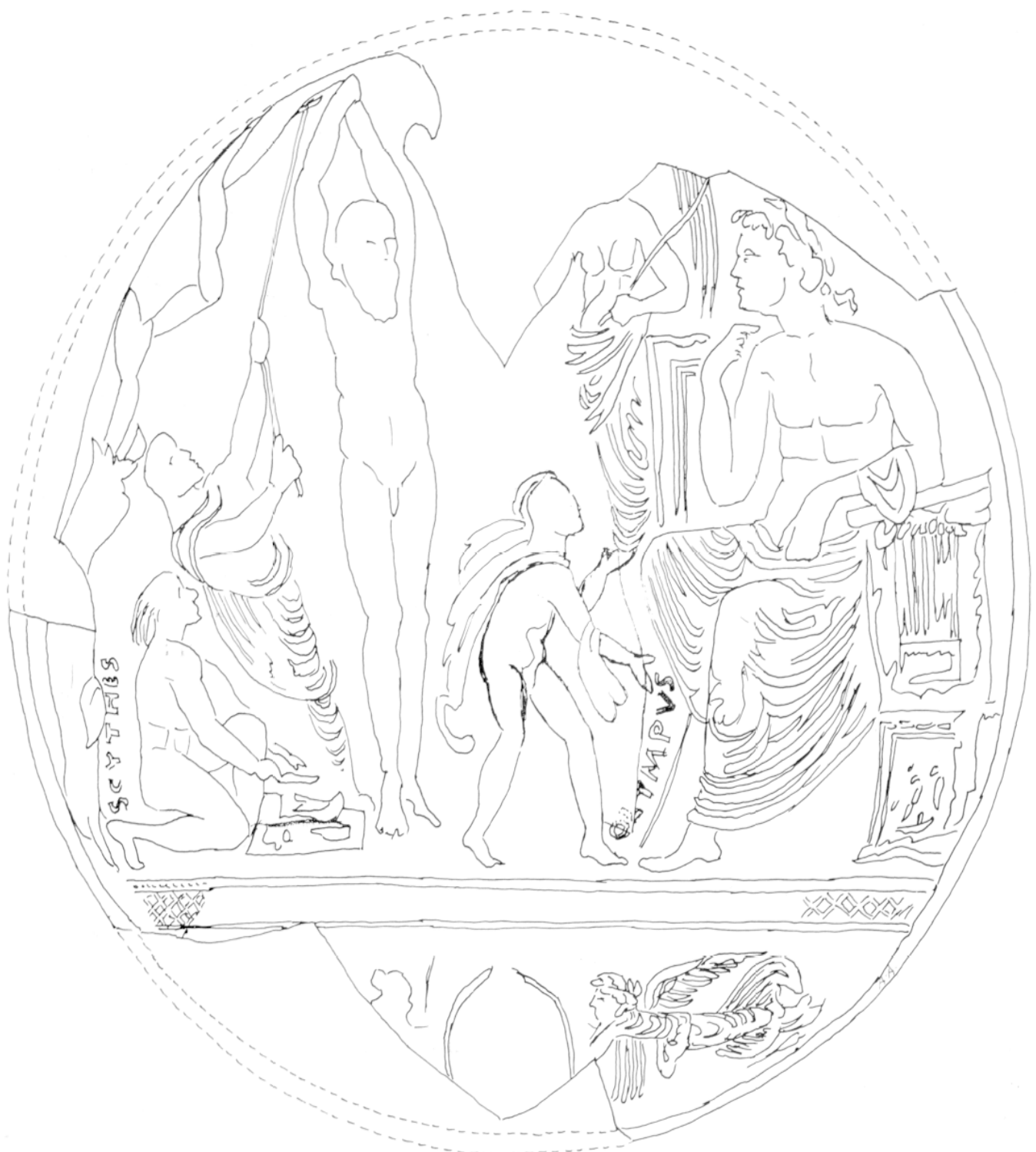

1 Le médaillon primitif restitué, environ grandeur nalurelle.

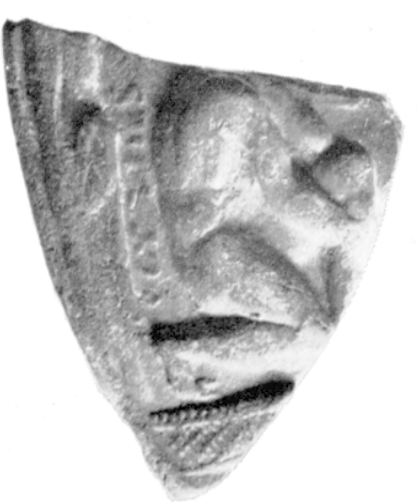

2 Fragnent du médaillon du supplice de Marsyas trouvé a Lyon. double de celle des autres personnages, lo dieu est assis sur un tròne à haut dossier earré dont le bord est décoré de perles. Il a le torse nu, le has du corps et les jambes couverts d'un manteau richement drapé at dont un pan vient retomber sur l'avant-bras gaurhe. Regardant Marsyas, il appuie le bras gauche sur la cithare, le droit replie, l'index tourné vers lui, comme pour répondre a Olympos: "T'el est le sort de qui s'attaque à moi ». La cithare, qui occupe l'extrémité droite, est posée sur un meuble bas, gauchi pour épouser la courbure du cadre et 
orné, semble-t-il, d'une scène à personnages analogue à celles qui décorent les coffres des chars des médaillons de Felix, et particulièrement celui de Cybèle. On ne peut qu'admirer cette magnifique image du dieu : elle constitue un tres beau décor.

Appuyé du bras gauche sur le sommet du trône, une femme de petite taille et qui, par un artifice de perspective, se trouve beaucoup plus haut que les antres personnages, contemple Varsyas. Privée de la tête et de la main droite, cette personne a, comme Apollon, le bas du corps recouvert d'une draperie aux plis tres serrés. Sa main gauche tient une haste très fine, et derrière son bras paraissent des lignes verticales qui pourraient figurer les rémiges de longues ailes repliées : il s'agirait alors d'une Vietoire, dans une pose analogue à celles qui encadraient l'autel fédéral de Lyon.

Aux six personnages assemblés au-dessus de la bande du sol, on ajoutera les deux qui ornent l'exergue : deux Victoires figurées horizontalement, en plein vol, les ailes ouvertes, long rêtues de robes à plis très mourementés. Elles tiennent un cartouche ovale qui occupe le centre de l'exergue rt sur lequel se lisent les trois premières lettres d'un mot qu'il est difficile d'interpréter : soit SUR soit SYA et quelques signes a la suite. 1)eux hypothèses peurent être proposées. S'agit-il du nom du céramiste lédosien Surillus qui, ayant repris le médaillon lyonnais, aurait effacé la mention figurée sur le cartouche initial pour la remplacer par sa propre signature"? Mais on s'étonnerait qu'il ait fait disparaitre un texte dont la présentation mème montre qu'il était essentiel pour la compréhension du sujet, alors qu'il pouvait placer sa signature en quelque autre point, sur le fond du médaillon. S'agit-il de la seconde moitié dlu nom de Marsyas, le reste etant inscrit ailleurs ? L'hypothèse n'est pas a rejeter car on trouve dautres noms de personnages ainsi fragmentes, mais il faudrait admettre que la première moitié était présentée d'une manière qui la mît autant en valeur que la seconde et, sur ce point, rien ne permet de justifier semblable hypothese. On attendra donc qu'une découverte nouvelle permette de répondre.

Voici. classés approximalivement dans l'ordre chronologique de la découverte, la liste des divers fragments du médaillon étudié.

A. - Fragment trouvé au $x_{10}$ siecle, sans doute à Lyon, conservé au llusée archéologique

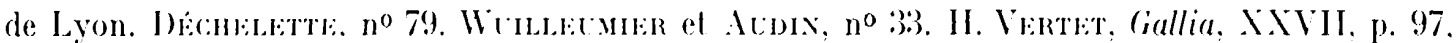
fig. 1 c (ici fig. 2 .

Vase a panse arrondie. Terre très rouge, sans engobe, relief non altéré. $14.47 \mathrm{~mm} ; 1.42 \mathrm{~mm}$ (hauteur du personnage de l'épaule au sol : $30 \mathrm{~mm}$ ). . De gauche à droite : cadre étroil, base du pin, légende verlicale : sGYTHLs, l'esclave privé de la tête, du pied grache et des mains; fragment de la bande du sol. - Alelier lyonnais, vraisemblablement de felix.

B. Relief d'applique sur vase entier trouvé à Lisieux "grand jardin", arant 1904. Conservé au Nusée de Rouen; .J. I)Échlistte, $n^{\text {os }} 12$ et 79.

Vase a panse ovoïde, forme 72 , orné de 4 reliefs d'applique (fig. 3 a, b). Terre fine à vernis rouge. Relief écrasé au démoulage, qui semble provenir aussi d'un moule usé, découpé sans forme précise et ne livrant que la moitié gauche de la scène. Hauteur totale du relief : $86 \mathrm{~mm}$; scythe, de la tête au genou : $35 \mathrm{~mm}$. de l'épaule au genou : $26 \mathrm{~mm}$; Narsvas : $8.3 \mathrm{~mm}$; Olympos : hauteur

4 H. VERTTT, op. cil., p. 101. 
METAIILONS I'APPLIOCE
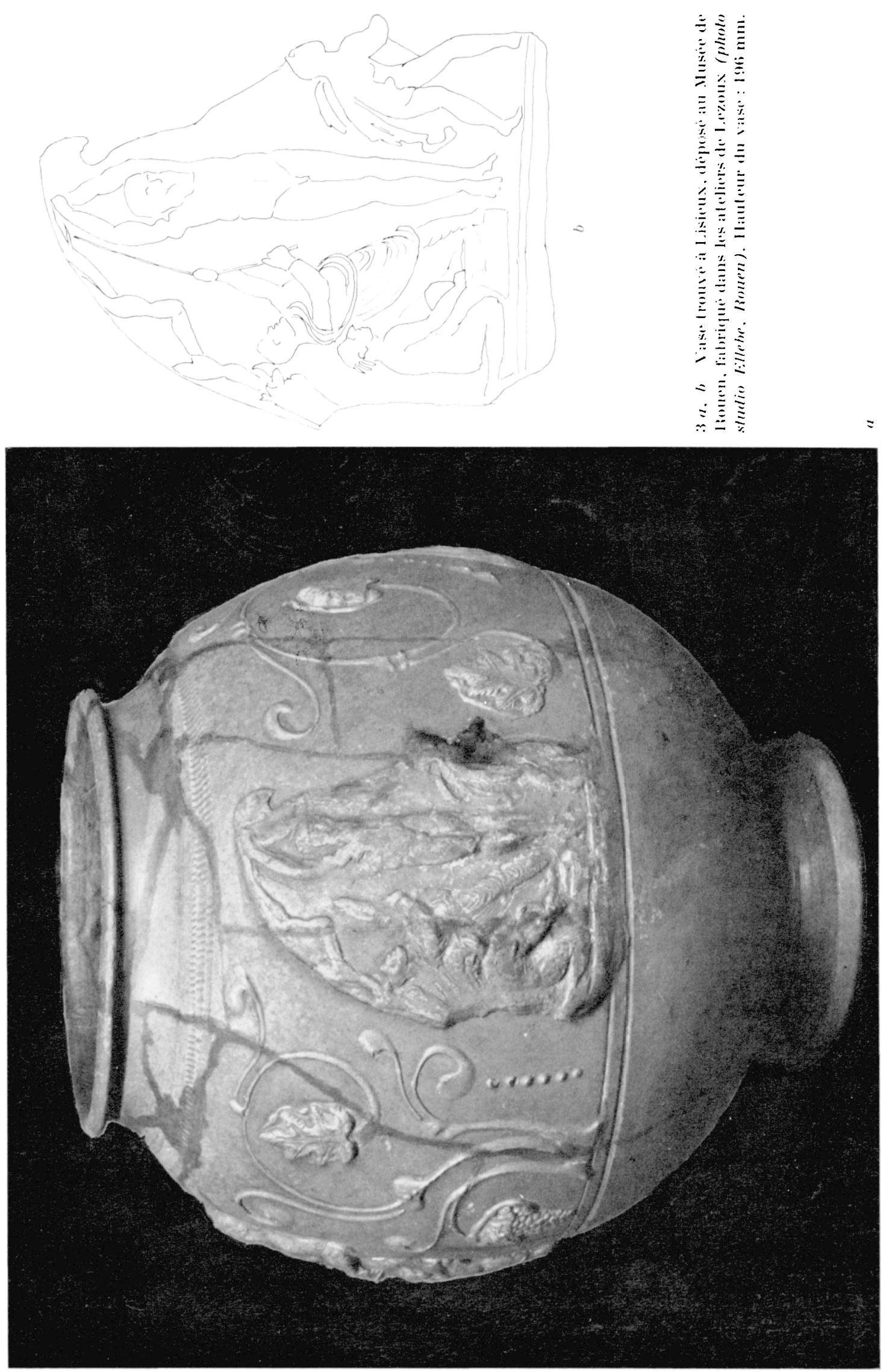


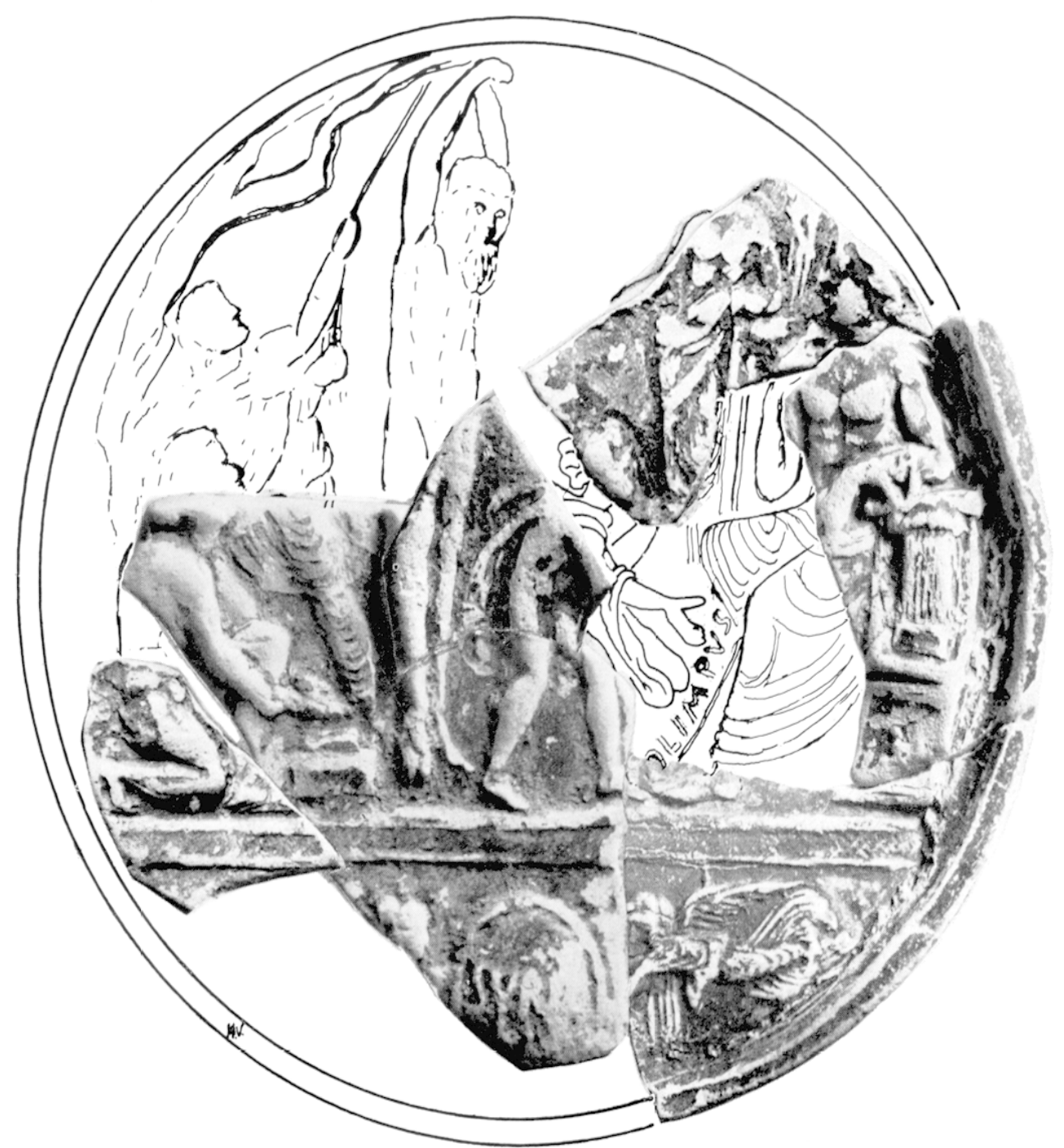

4 Gourde trouver a I.roux, montign des moreciux qui proviennent d'un mème monle, mais de plusieurs épreuves sepparies.

4:3 mm. Hituleur tolale du vase : $196 \mathrm{mmm}$. Les dimensions sont done sensiblement les mêmes que celles de la gourde trouvée à Lezoux, dont nous parlerons plus loin. Te gauche à droite : le haut du pin, le scythe. Ie lireur de corde. Marsvas entier. Olympos, sol réduil a un simple bourrelet, pats de cadre.

C. Fragment Irouvé en arril 19633. à Lezoux. dans les fouilles d'un dépoloir d'alelier bien daté (Trajan i. roule de Maringues. Conservé au Musée de Lezoux.

Fragment de gourde ; terre gris verdatre à couverte noire très érodée; h. $29 \mathrm{~mm} ; 1.32 \mathrm{~mm}$. .. On distingue la jambe droite el le has du torse du scythe. un élément non lisible de la légende el un morceau de sol: Irès forte réduction par surmoulages sucressifs. Bulle de moulage au plattre sous le genou.

J. Nème provenance que C. Ilusée de Lezoux.

Provient d'un flanc de gourde plat, forme (6). a ; terre grise à couverte noire érodete ; h. $34 \mathrm{~mm}$; 1. $: 31 \mathrm{~mm}$. .- Jambes de llarsyas sans les pieds et torse d'olympos. 

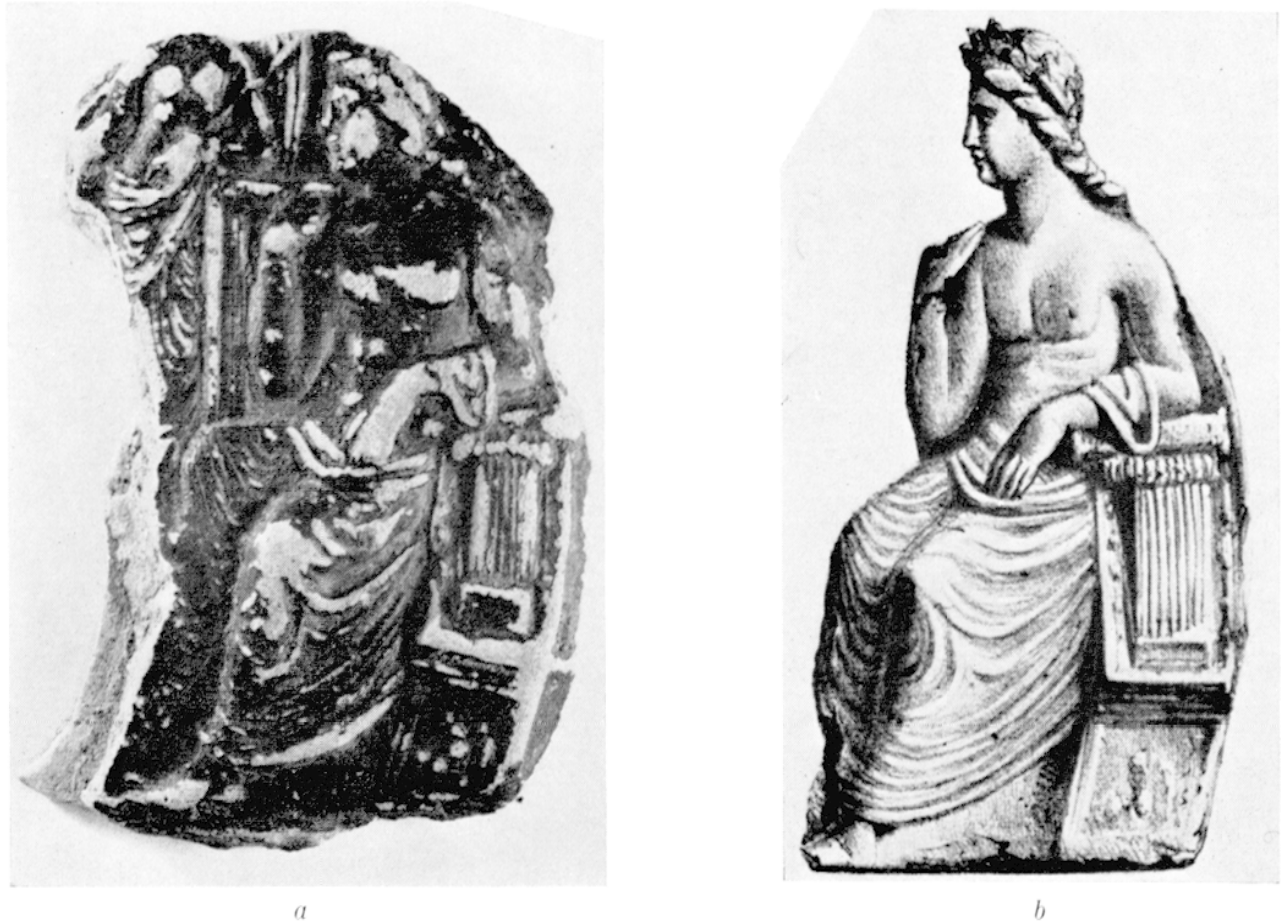

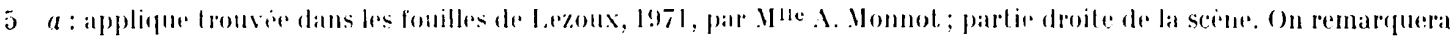

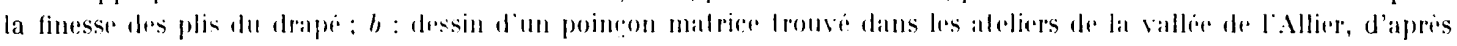
Tudol, colleclion de firnuriness en aryile, pl. 69.

L. - Mème provenance que Ci et I). Musée de Lezoux.

Provient d'un flane de gourde plat forme 6.3) a. Terre grise it couverte noire érodée ; h. 5i) mmo ; 1. $58 \mathrm{~mm}$. - De gauche a droite : lorse. bras droit et jambe gauche du sicythe jambe gauche et bas de la Lunique du tireur de corde, jambes de Marsyas, jambes d'()lympos. sious la bande du sol, le médaillon aver trois lell res lisibles el la tide de la Victoire de gauche.

F, G, II. Trois fragments jointifs trouves en juin 196i3 i Lezoux. mème lieu que les prérédents. Conservés au Vusée de Lezoux. II. Verter, Callia. X.XVII. 19699. lig. 1 a. p. 97.

Fragment d'un flane de goourde plat, forme 6.3 a, avec un grand morceau d'un cadre large et a fort relief et d'un còlé de la grourde. Terre grise a couverte noire érodée ; h. Lolale $98 \mathrm{~mm}$; 1 . fió) mm. Lal cithare, sur son socle, est haute de 3i) mm.

F : tête d'Apollon et divinité féminine privée de la tìte et du bras droit; tête d'Olympos. G : torse et hras d'Apollon. long morceau du cadre. II : sol avec le pied gauche d'Apollon, le pied gauche d'Olympos, la premire lettre : O, de son nom ; sous le sol, la Virtoire de droite, long morceau du cadre. (Les tessons C. D, E, F. (i. II sont réunis en un montage. fig. 4).

I. - Fragment trouvé en 1971, fouille de sauvetage de Ille Annie Ionnot, conservé au Yusée de Lezoux (fig. 5) a v ve le dessin fig. :) b).

Vase a panse ovoüde, forme 74. assez grand. Terre grise a couverle noire bien conservée, relief très nel; h. $80 \mathrm{~mm} ; 1.59 \mathrm{~mm}$. h. de la cithare sur son socle : $39 \mathrm{~mm}$; épaisseur de la paroi du vase : $3 \mathrm{~mm}$; épaisseur maximum du relief : 5 mm ; bulles de moulage au platre sous le pied d'Apollon. Apollon entier avec la cithare; devant lui. légende soulignée d'un trail : LIMPVS; le 0 initial inanque ; au-dessus du dieu. un personnage féminin acroudé a son tròne. sur le socle du tròne, une scène où l'on distingue au centre un personnage assis, devint lui un autre, plus petit, derriere 

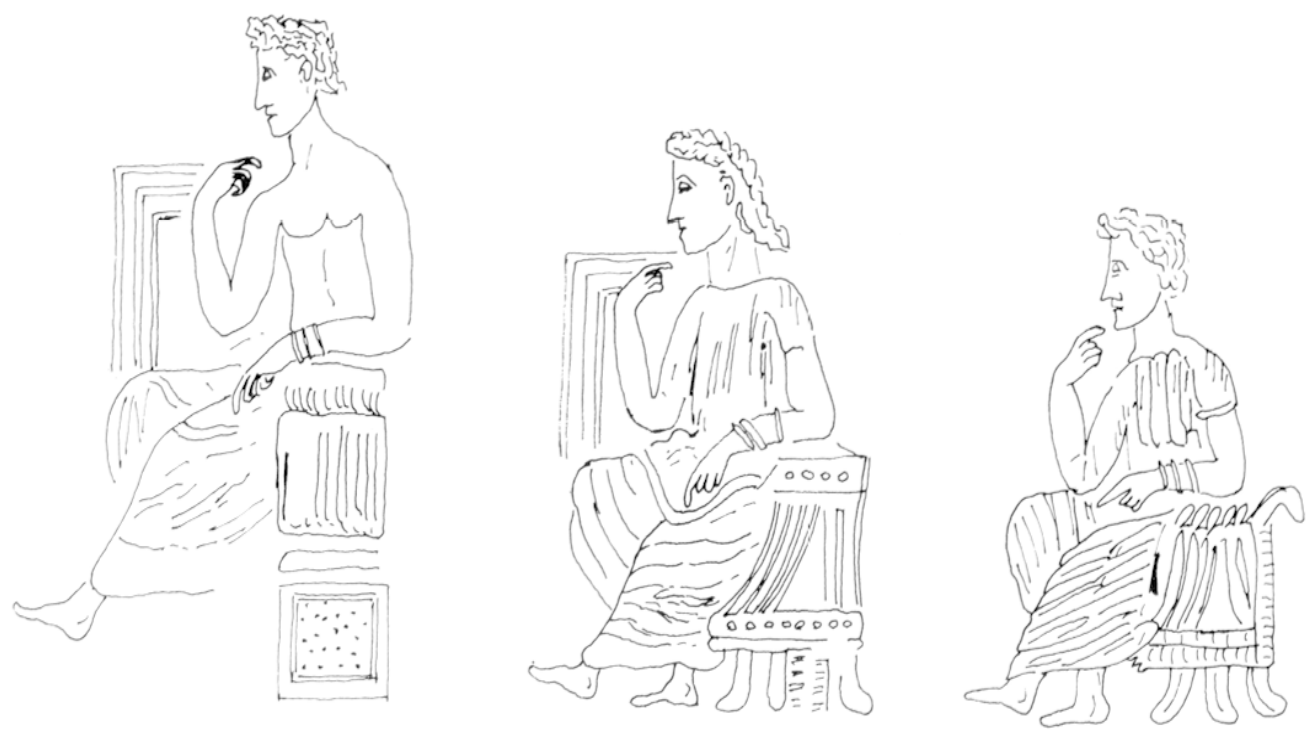

b

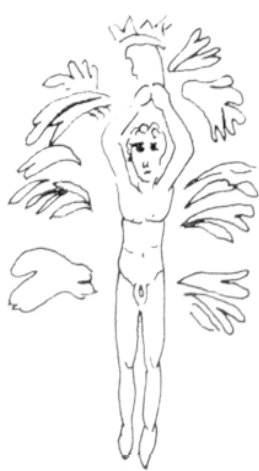

d

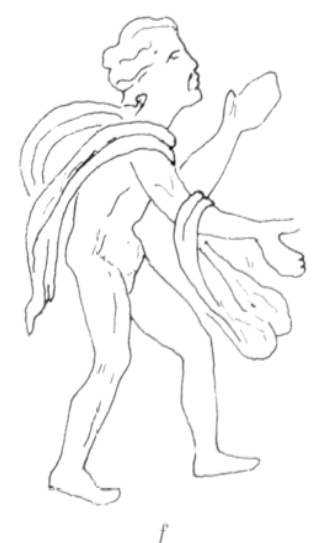

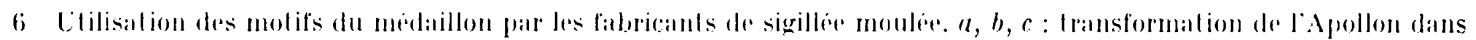

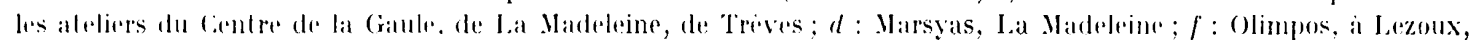
(he\% Bulrio, Caletus d'apris l'Inder d'oswald.

lai, un trosieme. plus grand. - Ce frament, mieux ronserve que tous les aulres lrourés jusqu'ici a Lezoux el d'une dimension plus erande, provient d'un surmoulage plus prorhe de l'original, probablement plus ancien.

Oulre ces neuf éléments, il existe huit rémplois de personnages isolés, préleves sur des vases

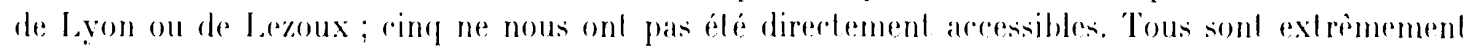
importants pour lhistoire du répertoire des appliques arvernes el de la sigillée.

J. Applique trouvée a Lezoux, dans le meme dépotoir d'alelier quue G, J). V. V. G. II, ronservi an Musée de lezonx. H. Ventet, Ciallia, op. cil. p. 97. fig. 1 b.

Fragment de vase a panse ovoüde forme 7.4 assez petit : lerre gris jaune a comverte noire; bulle de surmoulage au platre entre le menton et l'épaule: une autre au milieu du dos. Corps dus sirylhe. sims la libe.

K. - Ipplique trouve a lezoux ou a Clermont-Ferrand, conservée jardis au Musé de Moulins, aujourd hui disparue. J. DÉchets:Trs, II, no 9 b. 


\section{Sigillée - 1. Apollon}

L. - Poinçon matrice "trouvé dans les ateliers de la vallée de l'Allier", aujourd'hui perdu. Tcoot, Collection de figurines en argile, 1860, pl. 69. F. OswaLb, Index of Figure-lypes on terra

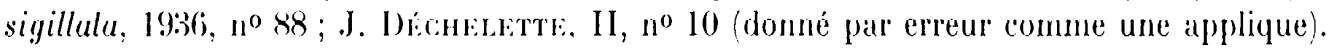

Le potier a relevé le seul personnage d'Apollon. Le relief épouse la forme arrondie du médaillon. Le dessin est probablement un peu embelli, comme ceux de cet auteur; le moulage a dù cependant etre pris sur un original en excellent état. Hauteur du personnage, selon Tudot : 79) mm (fig. i) b.

II. Mème motif ; ateliers de La Madeleine, dans le décor d'une forme 37 signé ALBIILIS's F (rétrograde). Epoque d'Ifadrien. F. Ors.max, dans Römisch-germanisches Korrespondenzblall, 1911, p. 91. F. Oswand), Index of figure-lypes on lerra sigillala, 1936, $\mathrm{n}^{\circ} 88,2$ (fig. 6 a).

X. Mrime motif ; le potier a modifié la cithare, qui est devenue un arroudoir du tròne (fig. (i) b).

L. Fö̈z:ı, Die Bilserschüsseln des oslgallischen Sigillala Mamufalituren, Bonn, 1913, n ${ }^{0} 469$, sur forme 37 , Bonn et Arentsburg, XII, 9.17 ; ateliers de Trives. Oswan.1), op. cil., no 89 ; ipocque d'Hadrien-Antonin.

O. - Vrime molif ; le potier a supprimé le còté droit du tròne. modifié l'arcoudoir gaudhe; probablement, évolution de .X (fig. $6 \mathrm{c}$ ).

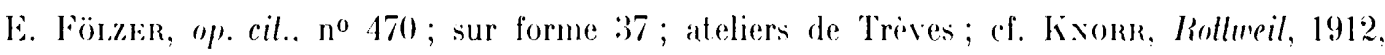

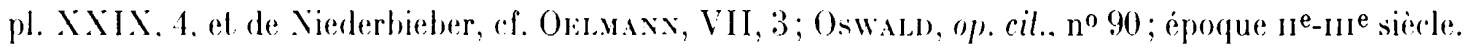

P. - Meme molif que (), mais un peu plus petit (surmoulage?) : FöLzER, no 471, sur forme:37. style de MAIAAVs, ateliers de Trèves; Fölzir, XVIII. 23.F. Oswand, op. cil., no 90.

\section{Olympos}

Q. Sur les morceaux de llancs de gourde, le personnage est incomplet, la seule figure entière que nous connatisions nous est donnée par la sigillée: J. I)ichlidette, no 398 ; F. Oswald, $\mathrm{n}^{0} 6$ 689) (lig...6 $6 \mathrm{~d}$,

Le molif apparail sur les vases de forme 37 , des Trajan che\% le potier BLTRIO, et il est encore employé sous les Intonins sur des productions du style de PATERNLs et chez C.LIIITLs (C.1 / I.IIT I II sur une forme 37 : T'. MAY, The P'oltery found al Sichesler, Reading, 1916, XXVII, 76).

\section{Marsyas}

R. I'n Marsyas, très réduit par les surmoulages et les reprises, apparait sur une forme 37 de l'alelier de La Madeleine: (Oswand, $n^{\circ} 72$. Le potier a imprimé tout autour du personnage des feuilles pour suggérer l'arbre ou est pendu le malheureux silène. Hadrien (fig. fo e). H. Rackix. Die Bilderschüsseln der Kastelle Saalburg und Zugmantel, dans Saalburgjahrbuch. VIII. 1934, IX, 1.

L'examen de ces fragments autorise les observations suivantes.

I'après celui de Lyon, le médaillon origrinal mesurait environ $140 \mathrm{~mm}$ de diamètre. Il avait été collé sur un vase à panse ronde. A-t-il été prélevé à partir d'un tel support par les potiers arvernes? Remettre à plat un sujet d'une telle dimension, sans altérer l'harmonie de la composition telle qu'elle nous apparaît à Lezoux, présente des difficultés techniques qui paraissent difficiles à surmonter. L'inverse paraît plus facile. En effet, 
on peut se demander si les originaux de certains sujets de Felix n'ont pas été concus comme des oscilla ou des flanes de gourde plate, puis utilises romme appliques sur des vases. Telle scene iliague du "combat pres des vaisseaux " nous est parvenue, comme "le supplice de Marsias" sur forme plate ot sur forme bombere et suggere la possibilite d'utiliser les deux formes simultanément ${ }^{5}$; mais il faut noter que ces reliefs plats sont anciens, que nous ne les connaissons qu'au début du ue sièrle, alors que les médaillons se fabriquerent jusqu'au nu On pourrait y voir un argument pour supposer que le document te plus ancien est te phos proche de l'original. Quant a la forme de la gourde portant ce médaillon, son originalité mérite description. Elle unit une fare tout a fait plane ot moulée a un dos qui ne semble avoir ebe qu'une feuille d'argile mince non moulée, à qui il arrivait de se deceller à la ruisson. Le coté, orné de deux cannelures, unissait les deux flanes. Il detait monté sur le moule mème et formait en outre, autour de la face déeorée, une saillie atteignant 3,5) mm. La gourde dont les fragments sont apparus a Lezoux en 19633 a seulement 2:3 mm d'épaisserur totale, ce qui réduit le vide interieur du récipient à $11 \mathrm{~mm}$ seulement cette gourde lédosienne est bien différente de la gourde lyonnaise dont les flanes, tous deux moulés, sont semsiblement. bombes. A ce type lyonnais, dont on trouvera plus loin un bel exemple dans le "vase Sallier" on attribuera le sigle fm (forme) $633^{6}$, la gourde lédosienne a parois plates portant lo sigle fm (j:3) i.

Le retrait subi par le médaillon original au rours dessurmoulages en ramena le diamètre, nouveau cadre compris, a 130 millimetres. Lopération entraina donc un retrait de plus de $10 \%$. Celte donnée ast importante rar, dans le recueil des médaillons d'applique rhodaniens, il avait été admis que certains sujets, et justement ceux qui, retrourés en plus grand nombre, avaient dû avoir le plus de suceès, avaient été édités en plusieurs modules. Les differences observeres pourraient done provenir de surmoulages, et non du modelage d'originaux de tailles différentes.

L'histoire du médaillon étudié ici ne s'arrète pass ainsi. Dans la suite, d'autres céramistes se contentèrent d'extraire du sujet, qui la belle figure d'A pollon, qui celle d'olympos, qui celle de Marsyas. Cés surmoulages ont-ils été prélevés sur des appliques lyonnaises ou sur des reproductions lédosiennes? Cne étude plus poussée pourra probablement nous le dire, mais déjà ils ouvrent une voie de recherche pour le problème irritant de l'origine des reliefs de la terre sigillée.

Au début du $\mathrm{II}^{\mathrm{e}}$ siècle, le répertoire arverne s'enrichit brusquement d'une grande quantité de motifs nouveaux, souvent très finement modelés. Joseph Déchelette avait supposé que le potier qui les a utilisés le premier, Liberlus, avait été en même temps un modeleur remarquablement habile, inspiré par la toreutique contemporaine . L'examen de plusieurs de ses personnages ne nous avait pas paru confirmer cette hypothèse ${ }^{8}$, et nous pensions plutôt que Liberlus avait été un ingénieux utilisateur de reliefs créés par d'autres.

5 H. Ventet, op. cit., p. 114, fig. 10.

6 Liste des formes des vases à appliques de la vallée du Khòne; H. Venter, op. cit., p. 108, fig. 6, et Réperloire de vases decorès au moyen d'un moule fabriqués à Lezour, dans Revue archéologique du Cenlre, XI, 1972.

7 DícinsteTtK, op. cil., I.

8 H. VintET, Hercule el Cerbère? Élude d'un molif de la sigillée par la mélhode pholographique, dans Revue archéologique de l'Est, X, 1952, p. 48-61. 
Yous avions déja pu établir, il y a quelques années, les preuves d'un transfert de reliefs d'applique de Lyon à Lezoux at de l’influence de cenx-ci sur la terre sigillée arverne10. Mais l'ensemble des documents ci-dessus constitue l'enchainement le plus remarquable que nous connaissions actuellement. Nous pourons le résumer ainsi :

a la fin du $1^{\text {er }}$ siecle vraisemblablement, ou au début du II $^{\mathrm{e}}$. un remarquable artiste lyonnais, Felix, morledr -- probablement en cire - des mélaillons diun tries beau relief. Ciertains sont plats;

les potiers arvernes les surmoulent of les utilisent romme flanes de petites gourdes assez originales. T'émoins en sont "le combat pres des vaisseaux", tire des tables iliaques,

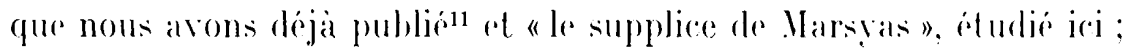

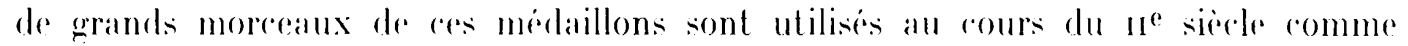
appliques sur des vases fom 7.4 (Trajan) et fm 7.2 Antonin, par exemple. la moitie du médaillon sur lo vase de Lisieux);

en meme temps, se produit un travail de dememberment. Les potiers surmoulent des personnages isolés (Apollon, le siegthe of les utilisent comme appligues sur des vases le faible dimension ;

à la mème époque. des fabricants de sigillée, travaillant dans les mèmes ateliers, auteurs vaisemblables des vases a appliques, relevent allssi des personnages boles of les emploient comme poincons matrices Apollon, Olympos). En ce domaine. re sont d"ingénieux artisans, non des créateurs;

ces motifs une fois entrés dans le répertoire de la sigillée y font une longure "arrière. On peut les suivere dans les ateliers du cientre de la Gaule jusquaux Antonins;

les atcliers de l'Est recoivent aussi les motifs créés à l'origine par Felix, les surmoulent, les modifient. T'émoins en sont le personnage d'Apollon, celui de Marsvas.

Yous arons done ici une filière précise, qui nous mène du potier lyonnais felix aux Arvernes Bulrio, Palernus, Calelus, à Albillus de La Madeleine, à Alpinus de Trèves.

Le sujet mème du médaillon du supplice de Varsyas, et surtout dans la version lédosienne des flanes de gourde, ne peut pas ne pas évoquer dans notre esprit, telle autre gourde qui porte le nom de son ancien possesseur, Sallier, d'Aix-en-Provence. Elle est conservée aujourd'hui au Musée de Saint-Germain et a été décrite par Déchelette, aux pages 307 et 308 des Vases ornés de la Gaule romaine'12. Nous ne décrirons pas en détail la face de cette gourde circulaire, qui représente le concours à boire entre Bacchus et Hercule. En revanche, l'autre, qui figure la lutte musicale entre Apollon et Marsyas, nous ramène à notre sujet.

9 H. Vанте, op. cit., Gallia, XXVII, 1969, p. 117-121.

10 H. Viertet, op. cil., p. 104 el 105.

11 H. VerTET, op. cil., p. 114-115, fig. 10.

12 On en trouvera aussi la photographie dans Gallia, XXVII, 1969, p. 112, a et b. 
Cette lutte se déroule en présence de tout l'Olympe. Du còté d'Apollon sont Diane, Mercure et une autre divinité. Du côté de Marsvas, une seconde déesse et Minerve casquée. A l'arrière-plan, Déchelette reconnaît Olympos et au premier "est assise Cybèle, dont le Silène n'a cessé d'être le compagnon fidèle ». Trois Muses, analogues aux Parques, arbitrent la lutte entre le citharède et le flûtiste qui, provoquée par le second, s'achérera par sa confusion et son tragique supplice.

Si le relief de Marsyas du vase Sallier n'est pas signé, l'autre face offre une banderole portant la signature APOLLINAR(is) CERA, celle d'un céramiste connu. L'analogie relative des supports, la parenté des sujets, l'un étant la conclusion de l'autre, suggéreraient qu'ils sont du même artiste. Toutefois le médaillon du supplice, surtout dans le fragment de Lyon, par le modelé, le dessin, l'acuité de la notation, l'aération de la composition, éroque singulièrement le style de Felix, auquel te recueil de 1952 avait attribué te médaillon. Le style d'A pollinaris offre quelque chose de plus pesant, de plus confus, de plus entassé. Il n'est done pas probable que les deux surres soient du mème auteur et nous nous réservons de revenir sur la seconde dans une étude consarréce a Apollinaris.

L'étude simultanée des médaillons lyonnais et arvernes offre enfin des viues nouvelles, sur le plan de la technique : alle permet de reconstituer des ensembles complexes at de les voir se fragmenter presfue sous nos yeux, entre les mains des fabricants de sigillée ; sur Ir plan commercial, elle révèle l'importane de Lyon comme centre de diffusion des schemes repris par les céramistes gaulois ot, du même coup, elle trahit l'influence de lyon sur le plan artistique, au plus lard des le début du $\mathrm{I}^{\mathrm{e}}$ siegle : enfin, sur le plan religieux, clle apporte des documents noureaux pour la ronnaissanere des religions de la Grece et de l'Orient et pour l'etude de leur expansion an Gialule.

\section{II. - MŕdahloNs d'Appligle et religions a mysteres}

Travaillant an classement des réserves du Vusée de Noulins, l'un de nous a en la bonne fortume de retrouver plusieurs fragments de médaillons d'appligue non encore signalés, bien qu'il s'agisse de trouvialles anciennes et que des ćléments des mèmes œuvres aient déjà été publiés dans le passé13. Il nous a paru utile d'étudier trois d'entre cux, d'abord dans le carle d'une recherche sur la céramique rhodanienne, mais aussi dans celui de la diffusion des religions à mystères orientales dans l'Occident latin. Ces premières démarches nous ont incités a clargir notre vision pour y inclure d'autres médaillons itroitement apparentés el à regrouper la documentation, peu exploitée jusqu'a présent, que la céramique apporte a l'etude des religions orientales en Gialule.

1. - Réserves du Musée de Voulins, provenant de la collection Lsmonnot. d'aprés le catalogue manuscrit de cette collection, mais une analyse de la pate. faite au laboratoire de Lyon. indiquerait plutot une origine arverne, sans que l'on puisse préciser encore l'atelier.

13 II. Verter, Observalions sur les vases à médaillons d'appliq̣ue de la vallee du Rhône, Gallia, XXVII, 1969, p. 93-133. 
Plus grande dimension du tesson : $85 \mathrm{~mm}$, appartenant à un vase, forme 7. Deux moulures supérieures sont encore visibles sous la lère, au-dessus du médaillon (fig. 7). ... Pàte rose, fine ; l'engobe, à l'intérieur et à l'extérieur, passe de l'orangé au brun, avec des reflets métalliques sombres dus à l'accumulation dans les creux. ce qui est normal sur ce type de vase. comme sur le lesson no 3. Epaisseur de la paroi, $2 \mathrm{~mm}$, de l'applique. $1 \mathrm{~mm}$ dans les fonds, $3 \mathrm{~mm}$ sur la roue du char. 1 l'intérieur paraissent les marques des ongles el du bout des doigls du potier. qui soutenait la panse tandis qu il appuyait l'applique à l'extérieur pour la faire adhérer. Le collage est habile mais rapide. Le pourtour de l'applique se trouve aminci et irrégulier. Les reliefs, assez flous, sont empàtés par les surmoulages successifs. Puslules de reproduction au platre, par exemple sur le bord de la jante. a l'intérieur de la roue et le long de la partie verticale du char. a droite. La premiere observation révele un surmoulage d'un original dont le potier n'a repris que la parlie centrale, la plus intéressante. négligeant le cadre qui le limitait. La seconde concerne les stigmates d'une reproduction au plàtre qui se manifeste par l'inclusion de bulles en relief dans les sillons reux. On a pu établir que l'usage du moule en plâtre était spécifique des ateliers de Lyon ${ }^{\mathbf{1 4}}$. Il est done vraisemblable que le médaillon original élait Lyonnais et nous reviendrons sur ce point. - Haul encore de $70 \mathrm{~mm}$, le médaillon, lel qu'il se présente sur le fragment de Moulins, est amputé de la partie inférieure el du cóté gauche. le droil élant, asse\% altéré. Le personnage principal est une femme debout sur un char auquel alaient attelés plusieurs individus, dont un seul est partiellement visible. On observe la forme ovale de la roue, qui devait compter huil rayons, dont l'un n'a pas été figuré. Des tètes apparaissent confusément a l'arriere de la scene.

Ce sujet est déjà connu par deux lessons ou s'observe également l'absence du huitieme rayon de la roue. J. de Wilte et J. Déchelelte en ont publié des dessins. Ie premier a été trouvé à Clermont-Ferrand (fig. 8), le second a Vichy (fig. 9j et a été conservé un temps dans la collection Rambert. Tous deux sont artuellement au Nusée de Saint-Germain. Comme celui de Moulins. ils ne reproduisent que partiellement l'original lyonnais. Celui de Clermont se limite à peu prè: aux mèmes parties que celui de Moulins, sauf que. à gauche, il montre deux des personnages lirant le char. Quant à celui de Vichy, il s'étale sur toute la largeur du médaillon original qui devait mesurer $89 \mathrm{~mm}$ de diamitre. Toutefois, la partie supérieure est brisée presque au ras du char, ne livrant à peu pris rien de la femme el de la foule, et l'on a volontairement escamoté l'exeroue, remplacé par une surface guillochée.

La confrontation des trois fragments permet de restituer presque totalement l'original. Le personnage debout sur le char est le principal. La femme est coiffée avec bandeau, chignon et ornement frontal très saillant, sans doute une fleur de lotus. Sa robe est couverte par un manteau passé sur l'épaule gauche et qui recouvre le haut du bras droit. De la main gauche, elle tient un objet arrondi, coupe ou patère, d'où semblent jaillir des branches assez informes. La main droite brandit un objet de forme pointue. En sautoir sur l'épaule gauche, parait une sorte de guirlande. Le char, arrondi devant, possède une caisse bordée d'une moulure à godrons et ornée de sept personnages en procession; la roue, ovale, n'a, on l'a vu, que sept rayons. Trois hommes, à tête rasée, au type accusé, tirent le char. I)eux d'entre eux, placés au-delà du timon, sont vêtus du cou aux genoux de tuniques plissées et de toges courtes, et celui qui est le plus proche du char, vu de face, porte au cou une bulla ronde tenue par un cordon. Le troisième, placé entre les deux autres, en avant du

1.1 Ibid.

15 J. w: Witrt, (iazelle archéologique, 1875, p. 93, pl. 25.

16 J. Díchistre, Les vases ceramiques ornes de la fiaule romaine, 1904, 11, p. 216-217. 


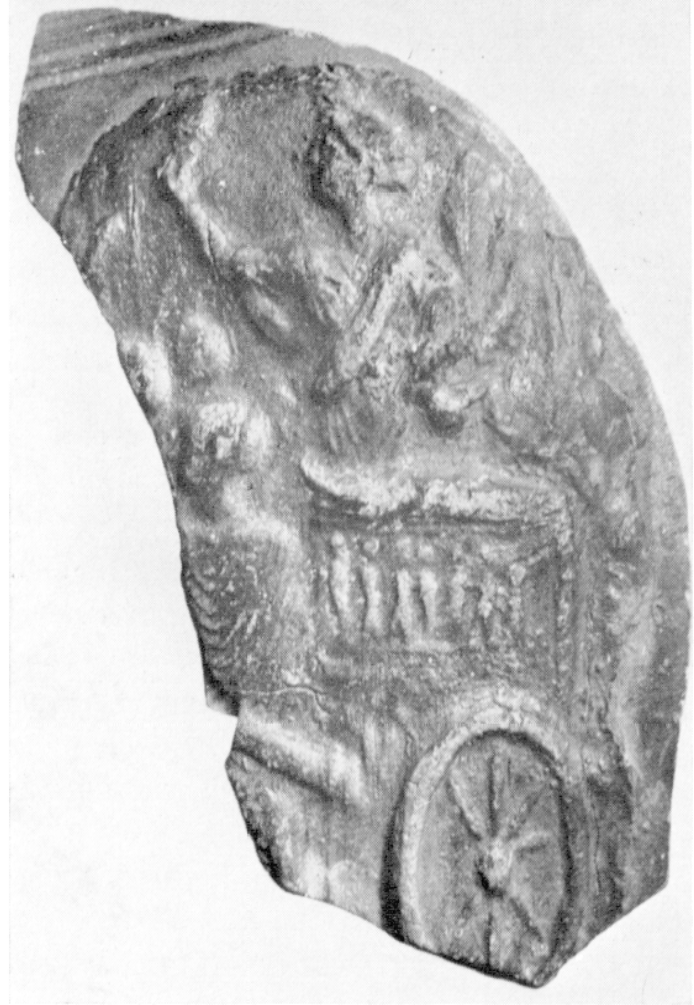

7 Le char d'Isis, de provenaner incomule Musíe de Moulins.

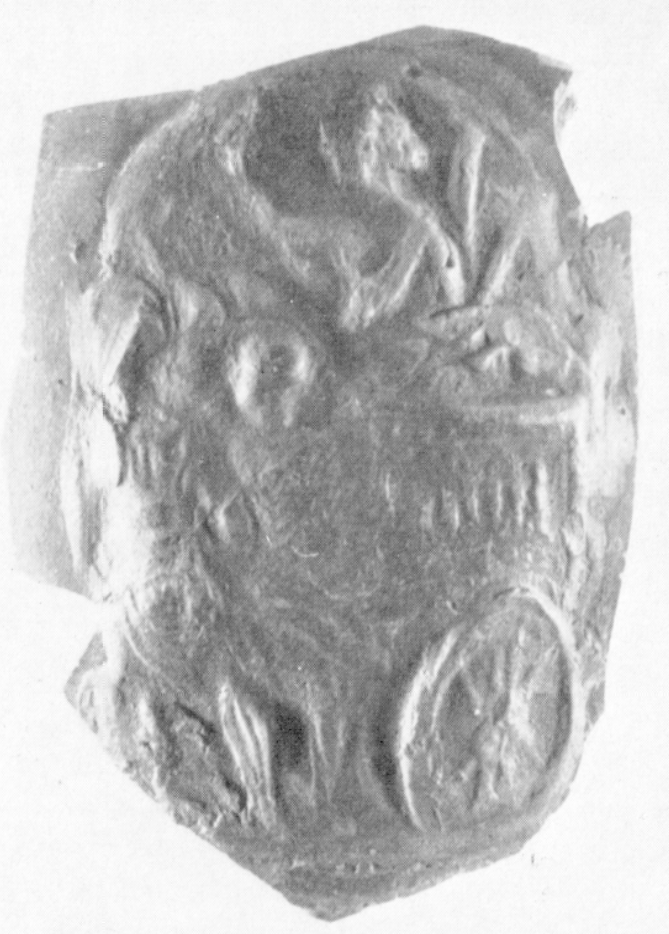

8 lo char d'Isis .lusion des antiquiles mationales de ilermont-Ferrand:

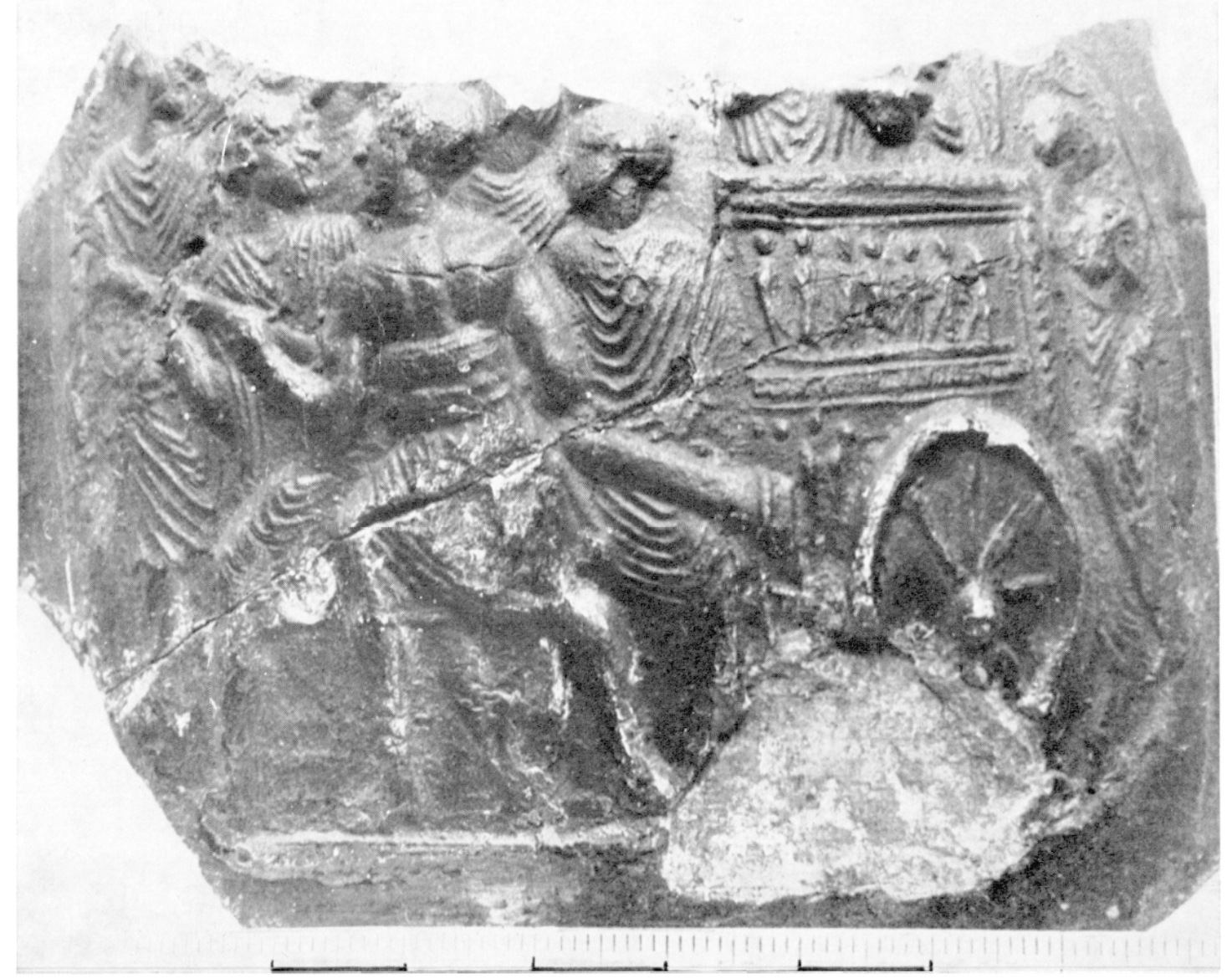

9 Le char d'Isis Musée des antiquités nationales de Vichy). 


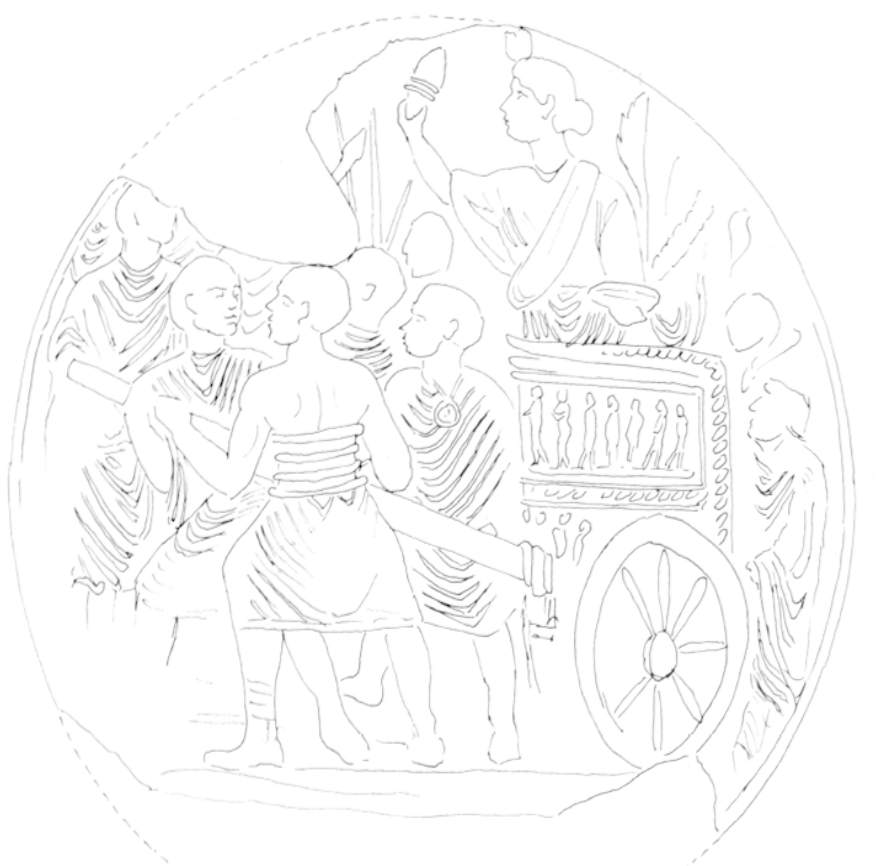

10 Le char d'Isis, restitution graphique.

timon et tournant le dos, est torse nu et porteur d'une tris large ceinture. Devant et derriere le cortege, deux hommes a pied sont vêtus de toges et celui qui est en tête tourne le visage vers le char. Au-dessus des hommes attelés au timon, des têtes assez confusément indiquées figurent une foule d'où émergent trois ou quatre hastes verticales. Ia scene est supportée par un sol que figure une cannelure saillante réservant, au-dessous, un exergue volontairement escamoté dans les trois fragments connus et qui devait porterun emblème ou, plus sùrement, une signature (fig. 10).

L'interpretation de rette scène, qui groupe une dizaine de personnes, a donné lieu à des hypothèses contradictoires. Pour de Witte et Déchelette, il s'agit du triomphe de Trajan. Le char serait tiré par des "prisonniers désarmés, mais ayant tous la tête couverte d'un casque ». Sur le char, derrière la Victoire, serait assis l'Empereur. En avant paraîtraient "d'autres figures confuses représentant la foule qui escorte le cortège impérial ». Cette interpretation soulève des objections. Les traineurs du char ne sont pas casqués, mais tête rasée, et surtout ils sont vêtus à la romaine. Il n'est pas logique, dès lors, d'y voir des prisonniers de guerre. Le personnage féminin désigné sous le nom de Victoire sera aisément identifié plus loin, et, à côté d'elle, on ne découvre, de Trajan, ni la place, ni la trace, mais seulement des formes indéfinissables, visibles sur le seul fragment de Moulins qui avait atteint un point d'érosion très avancé. Bien mieux fondée est la proposition de M. Andréas Alföldi ${ }^{17}$ : il s'agit d'un cortige isiaque. A ce titre, le tesson de Moulins est celui qui révèle le mieux la divinité. L'objet brandi par la main droite est plutòt une fleur de pavot qu'un sistre; du récipient arrondi que soutient la main gauche émergent un épi et, au-dessus, une fine tige qui serait celle d'un autre pavol; plus proche du coude, une forme renflée et sinueuse figurerait soit un épi, soit une corne d'abondance. Or sistre, pavot, épi et cornucopia sont les attributs d'Isis. Ajoutons que la déesse n'est pas laurée, comme le pensaient de Witte et Déchelette, mais qu'elle porte au front l'ornement très saillant que l'on distingue sur deux des fragments et que l'on retrouvera plus loin sur d'autres figurations d'Isis.

17 A. ALFöLII, Die alexandrinischen Göller und die Vola P’ublica am Jahresbeginn, dans Jahrbuch für Anlike und Christentum, 8/9, 1965-1966. 
2. - En faveur de l'interprétation isiaque, est décisif le rapprochement avec un autre médaillon, trouvé a Orange et offert, en 1917, par J. Pierpont Yorgan au Yetropolitan Vuseum of Art de $\mathrm{Tew}_{\text {York }}{ }^{18}$. Il montre un cortege dont le caractere isiaque est attesté par la présence d'un myste à tête de chacal figurant Anubis. Deux individus à pied, tête rasée à la manière des isiaques, lirent de la mème façon un char dont la caisse s'orne du même corligge de six petits personnages. La roue du véhicule affecte également une forme orale, mais à huit rayons. Il est suivi du mème logalus. Sur le char, Isis brandit un sistre ouvert, différent, semble-t-il, de l'objet figuré sur le fragment de Ioulins. Ia scène, qui comporte une dizaine de personnages, dont des porteurs d'enseignes, est soutenue par un sol semblable (fig. 11). Mais ici l'exergue est intact : il porte la signature Felicis cera. C'est celle de Felix, le plus fécond. le plus adroit. le plus célebre de tous les céramistes lyonnais, dont le style se caractérise par l'habileté des compositions, l'adresse des perspeclives, les justes proportions des grestes heureusement schématisés. La ressemblance el la symétrie des scènes figurées sur les deux médaillons, où le sens de la marche des cortigges est inversé comme pour établir des pendants, sont telles que l'on peut se demander si l'exergue du médaillon de .loulins ne portait pas également la signature de Felix, svstématiquement éliminée par les contrefacteurs. Enfin, s'il était besoin de confirmer l'attribution à Felix de notre médaillon, on rappellerait qu'il en existe encore un autre qui porte sa signature et figure un char dont la caisse est ornée de la mème procession : il s'agil du médaillon du Porlus alugusti, sur lequel on reviendra a la fin de cet article.

3 et 4. Wusée de Lezoux. Deux fragments numérotés 5is et 175, provenant d'un dépotoir de Lezoux, route de Maringues, juin 1963.3. Ils ornaient des vases de forme 74. Trois cannelures de faible profondeur sont visibles au-dessus du médaillon.

Premier fragment : terre rose pour le vase et sensiblement plus claire pour l'applique. Panse épaisse de $3 \mathrm{~mm}$, applique de 1 a $2 \mathrm{~mm}$ selon les reliefs. second fragment : terre de l'applique légèrement plus claire que celle du vase, dont l'épaisseur n'excède pas $2 \mathrm{~mm}$. L'état de conservation du premier est supérieur à celui du second. On observe, dans l'un el l'autre, la disparition totale ou partielle du cadre primitif (fig. 7). Premier tiers du ${ }_{1}$ e sircle. Le dépotoir, recouvert a cette époque, constituait un ensemble clos.

Le médaillon du char trainé à main d'homme par les isiaques s'est spontanément associé dans notre esprit à celui de New York. Une démarche analogue lie ce dernier, ou parait le dieu chacal Anubis, à un autre médaillon, d'un diamètre de $70 \mathrm{~mm}$, figurant la mème scìne et connu par ces deux fragments qui, se recouvrant partiellement, permettent d'en restituer la moitié supérieure. L'original dont ils procèdent est, à l'évidence, dans le style de Felix, à qui on peut l'atlribuer sans restriction. Le traité schématique et caricatural des visiges est parfaitement spécifique.

La scène représente un cortige marchant de gauche à droite, dont le personnage principal, situé vers la gauche était masqué par la tête du chacal Anubis. Autour de lui se groupent sept à huit personnages en vêtements à plis serrés. dont, pour la plupart, n'apparaissent que les têtes rasées. Derrière Anubis, l'un d'eux tient une haute palme; plus a droite et plus haut, un autre porte un objet qui pouvait être une palme ou une torche ; devant lui, un dernier, tête non rasée, pourrait être féminin ${ }^{19}$. Le rapprochement de ce fragment avec le médaillon de New York, signé de Felix, engage ì lui donner le même auteur (fig. 12).

5. - Egalement attribué aujourd'hui à Felix, un médaillon de $73 \mathrm{~mm}$ de diamètre est connu en trois exemplaires trouvés à Lyon. Il présente deux bustes affrontés : Sérapis a gauche, modius

18 P.Wuintecmer et A. Atron, Les médaillons d'applique gallo-romains de la vallée du Rhône, 1952, $\mathrm{n}^{\circ} 17$, donné par J. Díchelettr, op. cil., no 94, J. SAltri, Carle archeologique de la Gaule romaine, VII, p. $118, n^{\circ}$ 21, A. Al.För.DI, Tonmodel und Reiiefsmedaillons aus den Donauländern, dans Laureae Aquincenses Kuzsinszky, I, Disserlationes Pannonicae, 1I, Budapest, 1938, pl. LVIII, 4, A. A.FöI.DI, Die alexandrinischen Gïller..., pl. 10, 1. Curieusement placé sur un vase avec deux médaillons representant Thesée et Ariane. Metropolitan Museum of Art, n 17.194.870.

19 H. Vertet, Observalions..., dans Gallia, XXVII, 1969, p. 97, fig. 1, e et f ; ot p. 124-125. 

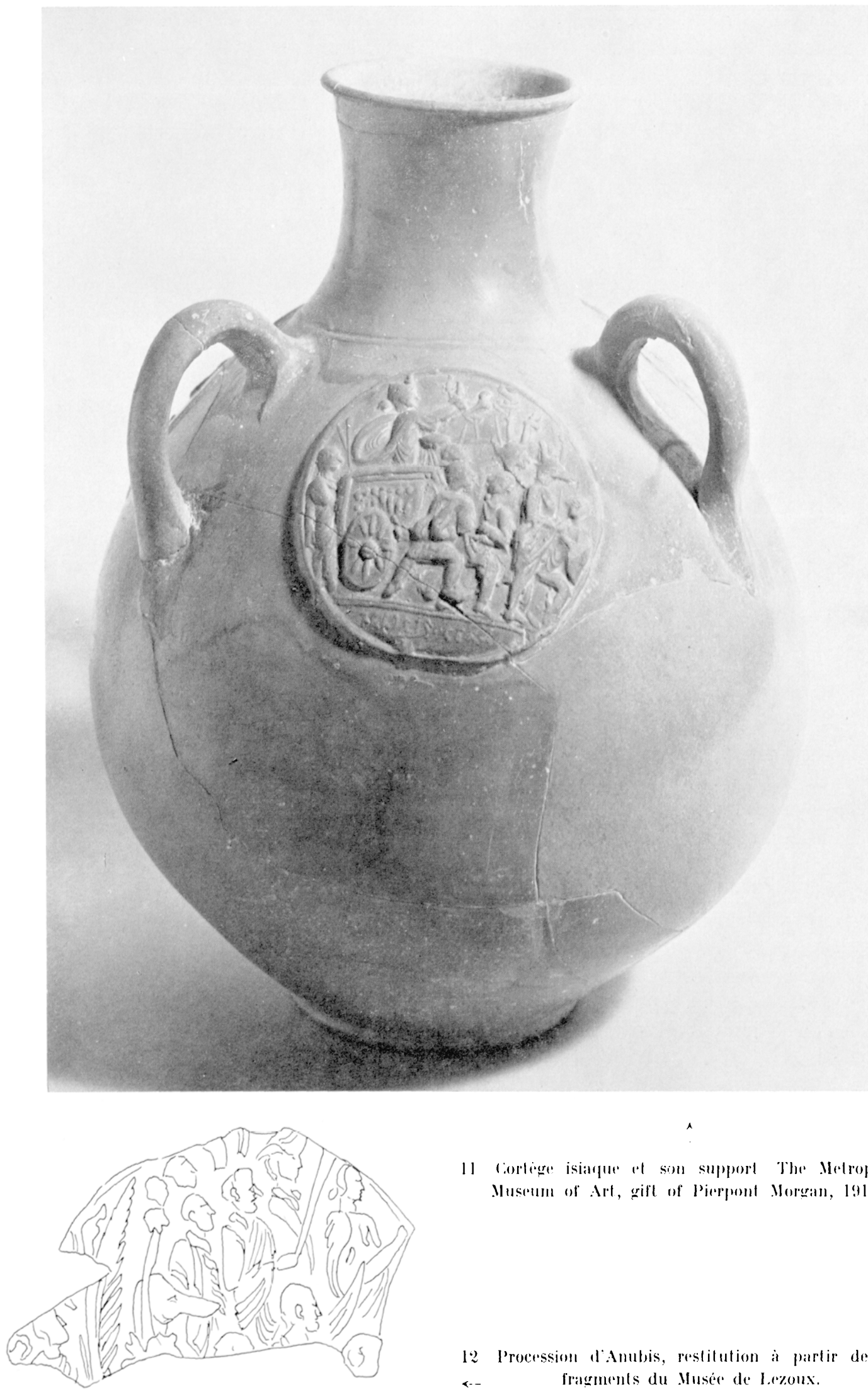

11 Corlege isiangue al son support The Metropolitan Sluserum of Art, gift of Pierpont Morgan, 1917).

12 Procession d'Anubis, restilution a partir de deux a- fragments du Musée de laroux. 
en tète, Isis a droite. Lintre eux, une fleur de parot est encadrée par deux épis. La déesse qui, comme sur le médaillon de Noulins, porte en sautoir sur l'épaule grauche une large guirlande, a te front surmonté d'un croissant de lune el d'un autre ornement emblématique indistinct, en forte saillie. Linsi ressemble-telle, trait pour lrait, a la déesse du fragment $n^{0} 1$. En exergue, huit petils personnages autour d'un autel sont traités de la même manière schématique et aiguë qui caractérise reux des chars de Felix ${ }^{20}$. Ce médaillon fut copié tardivement par un céramiste qui se contenla d'intervertir les deux personnages et d'ajouter, derriere Isis, un sistre fermé2.

6. - A Felix revient encore un médaillon trouvé ì Arles en 1943. W'un diamitre de $78 \mathrm{~mm}$, il montre une déesse dehout, tenant de la main gauche un plat et un panier, la droite tendant un sistre sur un autel. Jerrière elle, une palme. Le front de la déesse est chargé du mème ornement saillant déjà signalì22. Mr. Alföldi, plutòl qu'une Cérès, y veut trouver l'image d'Isis.

On acceptera donc sans réserve la thèse de cet auteur : le médaillon étudié ici sous le numéro 1 figure le corlège d'Isis, pendant de celui d'Anubis, œuvre du même artiste. L'identification est d'un vif intérêt car les deux œurres, et quelques autres, témoignent de la présence à Lugdunum d'une divinité qui, jusqu'ici, semblait s'effacer derrière Cybòle que les Orientaux de la ville tenaient en si grande révérence qu'ils lui avaient dédié l'un des plus grands sanctuaires métroaques de l'Oecident. Les Lyonnais n'ignoraient done pas la déesse égyptienne. On évoquera à ce propos la découverte faite, peu avant 1914, par Rogatien Le Vail, dans le quartier des Minimes à Fourvière, d'un Osiris funéraire en terre cuite (chouabti) que son frère, Bernard Le Mail, peu avant sa mort survenue en 1970, remit au Musée archéologique de Lyon.

7. -... Réserves du Nusée de Ioulins. Provenance inconnue (vraisemblablement Vichy, Clermont-Ferrand ou Lezoux. Plus grande dimension du tesson : hauteur $28 \mathrm{~mm}$, largeur $25 \mathrm{~mm}$. Décorait un vase de forme 74 dont il reste trace de la zone guillochée inférieure.

Pàte chamois clair de texture fine. légèrement micacée, engobe brune, presque noire à l'intérieur et, a l'extérieur, plus claire sur les parties saillantes de l'applique. Épaisseur de la paroi $2 \mathrm{~mm}$, de l'applique 1 i $: 3 \mathrm{~mm}$. - - Tous les détails sont nets, l'objet est sorti d'un moule en bon état. Pustules de surmoulage au plàtre dans les plis du vêtement. . Première moitié du II $^{\mathrm{e}}$ siècle.

bans ce second fragment de Ioulins, il s'agit, la encore, d'un surmoulage dépourvu de cadre et dont l'exergue a disparu au profit d'une partie guillochée. Le sujet est un personnage marchant. vers la gauche et suivant un char dont la roue est partiellement visible. Il est vêtu d'un long manteau bien drapé et porte des demi-bottes pointues «à la poulaine» (fig. 13).

Bien que le premier examen fasse songer à notre cortège isiaque, il n'est pas possible de superposer les dessins, encore moins d'identifier le personnage très largement étalé, avec celui qui suit le char d'Isis et qui, coincé entre cadre et roue, est comme étiré en hauteur. Il s'agit donc d'un nouveau médaillon à char, dont il n'est pas évident qu'il soit de Felix. Malgré cette incertilude, il ne nous a pas paru légitime de le néoliger.

8. - Réserves du Ylusée de Youlins. Provenance inconnue (vraisemblablement Vichy, Clermont-Ferrand ou Lezoux). Plus grande dimension du tesson, $50 \mathrm{~mm}$ en hauteur et en largeur. Décorail un vase de forme 74 . 
La pàte du vase est rougre. celle du médaillon, jaune. Engobe brun foncé à l'intérieur et à l'extérieur. Épaisseur de la paroi, $2 \mathrm{~mm}$. de l'applique. $4 \mathrm{~mm}$ dans les parties massives du corps du grand personnage et de l'enfant. A l'intérieur apparaissent. comme sur le $n^{0}$ l. les traces des ongles et des doigts du potier. Les reliefs, flous, se sont empàtés lors des surmoulages successifs. Pustules de reproduction au plitre par exemple au-dessus du poignet gauche du personnage central. Premiete moitié du II $^{\mathrm{e}}$ siècle.

Provenant du centre du médaillon original, re fragment ne comporte pas trace du cadre, et sa forme losangique en réduit encore la surface visible. Il montre a droite un homme en bonnet phrygien tendant une patère a une femme. Entre les deux apparait la tèle d'un enfant; au-dessus, un cortege marche vers lil gaturhe (fig. 14 .

list-ce un hasard si ce médaillon. dont le style est semblable en tout a celui du médaillon isiaque, se retrouve sur les deux vases du Nusée des antiquités nationales qui assorient déja les deux sujets? On ne saurait l'admettre et l'on est conduit à penser que c'erl ains ćramistes marginaux ont fabriqué en série des vases portant les deux médaillons.

La signification du sujet est largement ietendue par confrontation aver les deux appliques du Iusée des antiquités nationales ef spécialement avec colle de Vichy, de forme approximativement carrée, de $60 \mathrm{~mm}$ de haut sur bo de large, et qui se présente dans un état de fraicheur permettant de déceler nombre de détails invisibles sur les autres (fig. 15). La scène émane d'un original dont le diametre atteignait ou dépassait 7a) $\mathrm{mm}$, rt dont les surmoulages, par lesquels il est connu, ont également escamoté le cadre et l'exergue. Le personnage principal est signalé par sa taille hyperphysique. Debout dans la partie droite du médaillon, regardant à gauche, il est jeune, imberbe, coiffé d'un bonnet phrygien richement decoré d'une rangée de perles autour du front et d'une autre sur l'arête supérieure dont la pointe retombe dans le dos. Sur une tunique à longues manches, blousant à la taille et descendant aux genoux, il porte une courte chlamyde fixée par une fibule ronde sur l'épaule gauche. Les jambes sont gainées dans des anaxyrides, longs pantalons à l'orientale, serrés aux chevilles. Il est chaussé de bottines portant trois godrons sur les côtés. De la main droite, il éleve une branche dont se détachent des feuilles lancéolées. De la gauche, il tend une patere à une femme debout et lui faisant face. Ie taille beaucoup plus petite, elle est vêtue d'une tunique talaire. Sa coiffure est faite d'un rouleau encialrant le front. Elle tend la main droite, seule visible, pour saisir quelque chose dans la patere. Derrière elle, d'une stature plus élevée mais moindre que celle du Phrygien, un homme en toge est debout, la tête levée pour regarder le visage de celui-ci, le bras droit le long du corps, le gauche caché. Entre la femme et le Phrygien, un enfant de face, vêtu d'un long manteau flottant, danse et, des deux mains, brandit des fleurs en une attitude d'extrême jubilation. Seuls ces quatre personnages, placés au premier plan, portent sur le bourrelet formant sol, témoignant par là qu'ils sont les principaux acteurs de la scène.

Les comparses ne sont pas sans intérêt. Derrière l'Asiate paraissent deux personnages plus petits. Celui de droite, en pied, est vètu d'une toge courte et tient un fouet de la main gauche. Au-dessus des deux hommes, un registre rectangulaire, orné d'une rosace faite de petits points, pourrait être un bouclier dont le porteur ne montrerait que son torse nu, aux larges pectoraux. A l'arrière-plan, comme étranger à cette scène, un cortège marche de droite à gauche. Parmi les gens qui le composent, est placé bien en vue un individu revètu d'une tunique dont la manche gauche, plissée comme celle des autres figurants, 


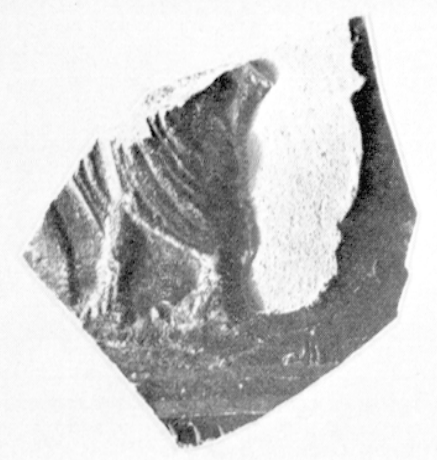

13 Prowession Musion dre Monlins.

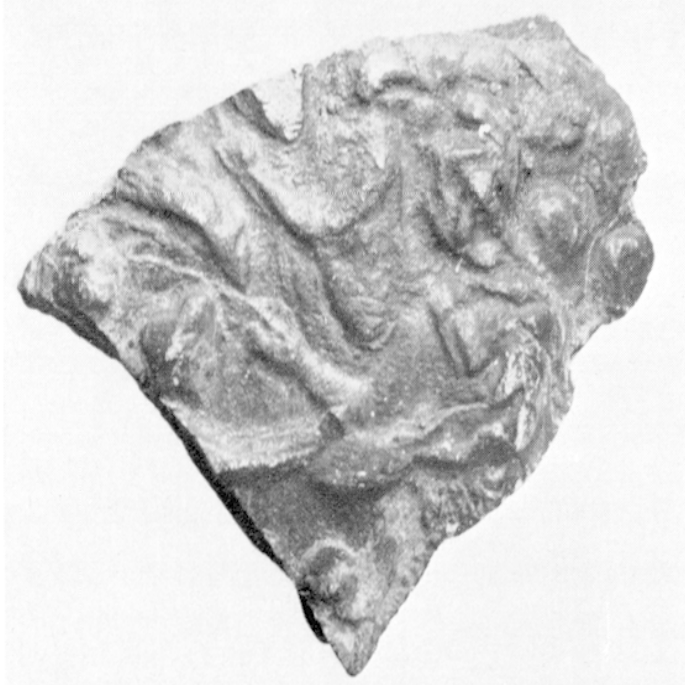

11 Nllis Mused ar Moulins. >

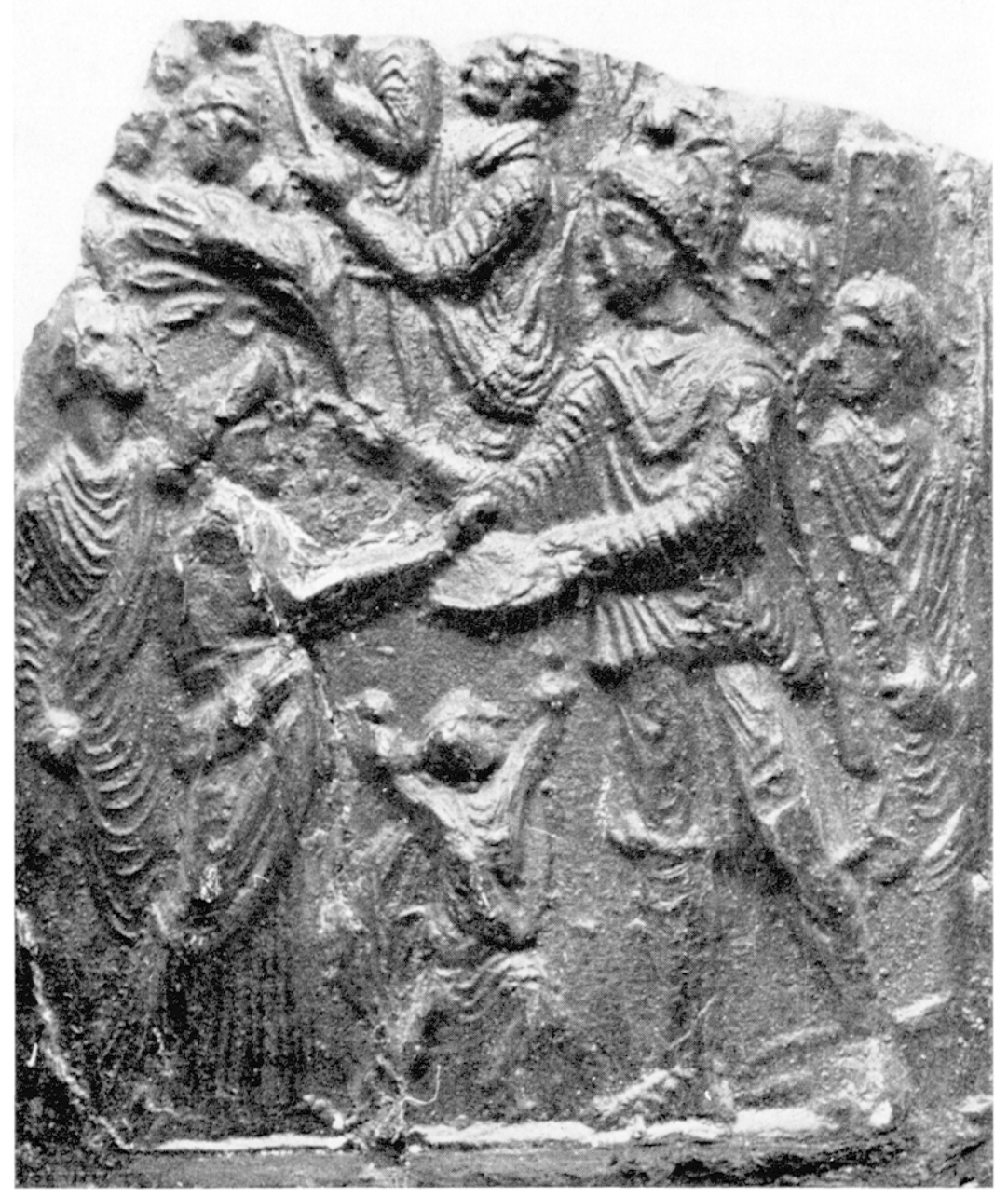

15) Nllis 11 son cortige Ilusio de Moulins?

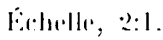

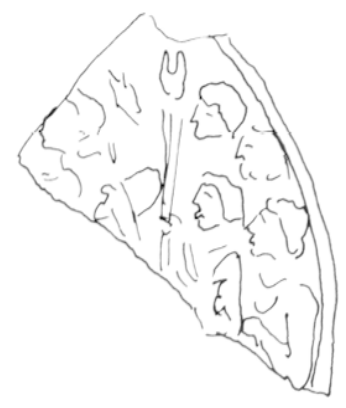

16 Fragment du corlege avec torche Musie archeologicyue de lavol. 
laisse l'avant-bras nu. Ln manteau retombe sur la hanche gauche. De la main visible, il tient une haste mince dont l'extrémité a malheureusement disparu. Son regard est levé vers l'objet qu'il brandit ainsi, objet léger car son bras est presque étenclu et le support de faible diametre. A sa droite, un autre personnage tient, lui, son bras presque replié, sans que l'on puisse deviner ce qu'il tenait à la main. En arant, paraît un homme dont la tète est soit casquée, soit couronnée d'un bandeau. A l'exception du Phrygien, de la femme et de l'enfant, tous les participants ont les chereux à la Titus, ce qui les distingue des isiaques rasés du premier médaillon. Restent inexpliqués les points qui se trourent en avant du visage de la femme et la patère portée par le Phrygien.

Le fragment trouvé a Vichy, aussi bien que celui du Musée de . Noulins, porte la trace d'une réparation ou d'une modification maladroile de la parlie centrale. Elle affecte surtout le bras de la femme. qui est informe et exagérément long au point de modifier peut-être la signification de son geste. Elle s'étend aussi au visage de la femme et à celui de l'enfant, qui sont déformés et caricaturaux, et a pu également intéresser la palire et la main qui la tient. La rareté des bulles en cette zone suggère qu'elle est relativement récente. Il se peut que le moule ait été brisé : un bourrelel. provenant d'une fente, traverse en biais le médaillon, passant au-dessus du bonnet phrygien et descendant jusqu'au bas de la robe de la femme. Il est d'autres exemples de moules de médaillons brisés et recollés, par exemple dans "le combat près des vaisseaux "23, et même de moules de vases sigillés qui, brisés, furent utilisés sans que le potier ait pu assujettir exactement les fragments assemblés. C'est probablement à l'occasion d'un tel accident que sont intervenues les maladresses qui affectent ce médaillon. En outre, il faut se défier de l'abondance des pustules qui, par exemple, fourmillent dans les plis du manteau du Phrygien, au point que, au premier coup d'œil, on penserait à un décor de perles.

L'interprétation de cette scène, d'ailleurs incomplète, soulève des difficultés. On écartera sans hésiter celle de de Witte et Déchelette, qui prolonge leur exégèse du premier médaillon. Celui-ci figurant, à les croire, le triomphe de Trajan, le second représenterait le roi barbare Parthamaspate, vaincu par l'empereur et apportant un rameau d'olivier à l'impératrice Plotine qui accompagnait son époux dans l'expédition contre les Parthes; le personnage debout derrière elle serait Trajan lui-même. Cette interprétation ne résiste pas mieux que la première à l'examen. Déjà, il est pour le moins étonnant qu'on ait réservé au vaincu une taille supérieure à celle du vainqueur. Ce n'est d'ailleurs pas le rameau mais la patère, que le Phrygien tend au personnage féminin. Au surplus, l'empereur devrait paraître en costume militaire, peut-être même à cheval, pour recevoir la soumission du Parthe. Enfin, comment expliquer la présence et l'attitude de l'enfant qui, malgré sa petite taille, est un élément essentiel de la scène ? Il resterait également à justifier le cortège avec le hastifère qui compose le fond du tableau. Et nous ne parlons pas du porteur de fouet.

Ayant écarté cette hypothèse, fondée sur l'apparentement des deux médaillons qui ornent le même vase, on se défiera de la propension à lier systématiquement l'un à l'autre, et, la signification historique rejetée, à chercher dans le second une nouvelle scène isiaque.

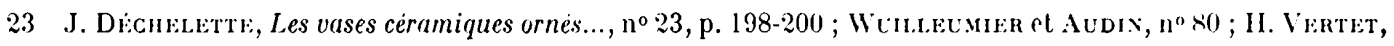
Observalions..., p. 115 et fig. 10. Cassures visibles sur le dessin, comme sur les photographies. 
On pourrait, certes, penser que l'enfant rst Horus, tout a la joie de la résurrection de son père Osiris, auquel Isis vient de rendre la vie. Mais si la taille du personnage a bonnet phrygien le designe comme un dieu, ce dieu n'est pas égyptien : vêtu a l'orientale, porteur du bonnet et des anaxyrides, il ne peut être qu'un dieu authentiquement asiatique. Là, encore, se présente une alternative. Deux divinités portent le bonnet : Mithra, le parthe, et Attis, le phrygien. L'aspect du dieu n'est pas en faverur de la première hypothese: Vithra porte le plus souvent un manteau ronstelle at rarement des anaxyrides. In autre retail de la scène sera-t-il plus révélateur? Au sommet paraît un hastifirere. Sa pose evoque singulièrement celle d'un personnage d'un autre mérlaillon attribur a lelix at ou le porteur tient une torche fig. 16). Cartes. ce médaillon, d'un diamètre beancoup plus réduit, et ne placrant pas la torche an mème condroit, n'est pas superposable à celui qui est étudir iri. Au moins fournit-il une raison d'interpréter le geste du personnagere. Transposí dans le cadre de la religion mithriaque, le porteur de torche ferait songer a l'un des deux dadophores, Cauties et Cautopates, qui encadrent. le dieu taurortone. Toutefois, la reggle est que l'un des deux tient une torche leveer, l'autre abaissée, symboles astraux, et ici aucun n'offre l'attitude du second, tandis que celle du dieu qui serait Mithra ne rappelle en rien celle de l'egorgeur du taureau rituel.

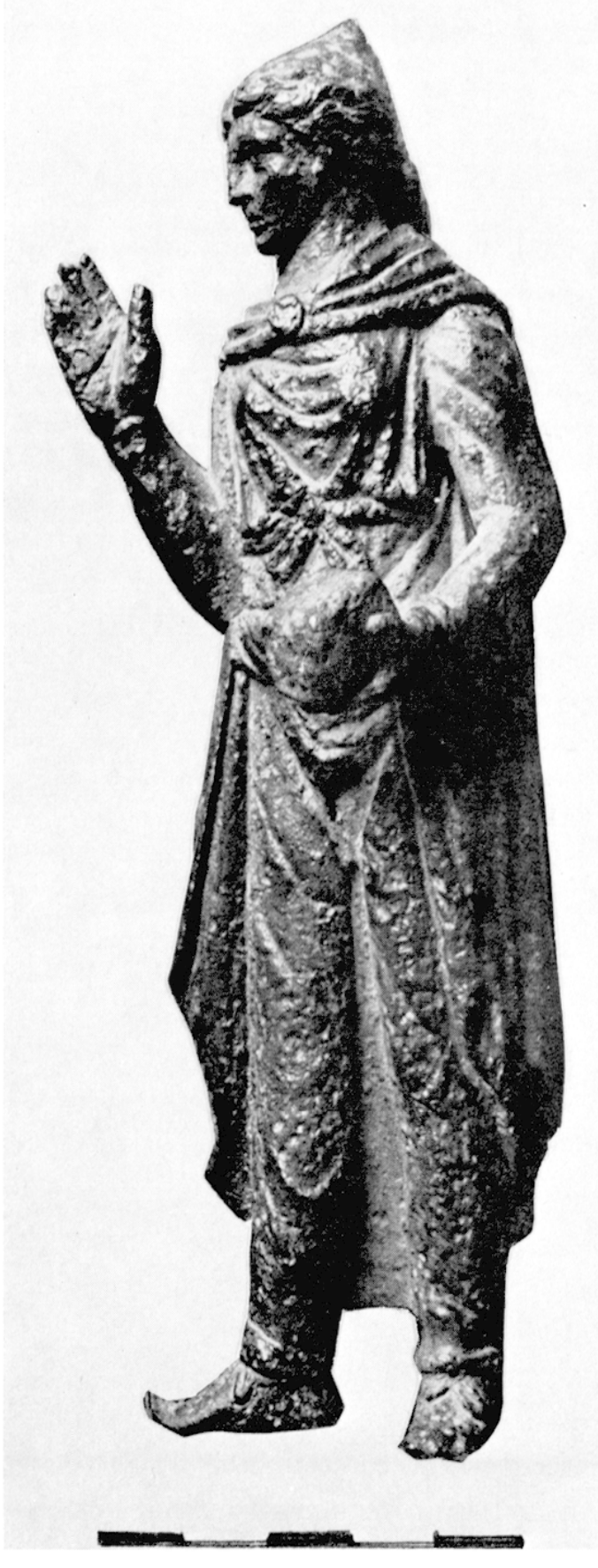

17 Attis, statuelle an bronze (coll. I.alournerio:

Exclure l'hypothese perse, ou parthe, qui nous aménerait a une date un peu basse, implique que la phrygienne s'aceommode beaucoup mieux des détails de la scène. Le personnage de taille hyperphysique serait Attis, fils de Cybèle, la Girande Mere de Phrygie. Il n'est d'ailleurs, pour s'en convaincre, que de la comparer à la belle statuette conservée 
dans la collection Latournerie et provenant raisemblablement d'Ampurias. Ce bronze figure le dieu, ou son prètre, dans le mème costume et dans une position qui n'est pas sans analogie aver relle du dieu du médaillon, à cette différence prese qu'il ne tient pas une patiore mais un coffret (fig. 17).

Quant à l'identite de ceux qui font face à Attis, on songe dabord au prètre at à la pretresse de Cyblete. Efferdivement, l'autel taurobolique lyonnais de 197 rite nommément prètre et prètresse.25. Ca noest pas l'unique hypothese possible. Si, sur plusieurs autels trouvés à Lỵon, un seul mentionne la prètresse, trois citent d'autres femmes dont le ròle semble plus important. Ce sont, en 194, Aufustia Alexandrina et Sergia Parthenopea ${ }^{26}$, an 197, Septicia Valeriana et Optatia Siorata auteurs du vou qui motiva le sacrifice. Lit pour montrer que le nombre de deux nétait pas impose pour tels voux, sur un trobisieme autel, la dédicante ast la seule Billia Veneriate. On peut au moins proposer que, sur notre mérlaillon, le personnage féminin soit la dédicante, aceompangé d'un personnage maséculin qui serait un re-dedieant. Derrière le dieu, le porteur du fourt serait l'arehigalle, dont le nom n'est jamais oublie sur les autels lyonnais, et dont .l. G. Richard nous a rappele qu'il avait justement un fourt pour attribut. Le myste porteur de haste serait soit un hastifere, dont la presener est rffectivement attestee dans le cortige métroarque, soit un dendrophore, porteur du pin sacré qui voyait la passion d'Altis. La mutilation du médaillon ne permel pas de prendre parti entre les deux hypotheses. Pour courrir entierement la scene figurée, il reste à décourrir l'identite de l'enfant qui marque sa joie all premier plan. Rien rlans le rituel des fàtes equinoxiales de cybele ne paraît justifier la presence de ce personnage, qui ne peut ètre que symbolique. (On est conduit à penser qu'il exprime le nom mème et la signification de la fête des Ililaria qui, le 2.) mars, consacrait la resurrection l'Attis dans un climat de liesse pour tous les dévots de l'eléenne, dont elle terminait le arele annuel. Aucune proposition n'expliquerait mieux, semble-t-il, l'attiturle quelque peu insolite de l'enfant.

Certes, dans ce médaillon lié à la théologie de Cybèle, la déesse elle-mème est absente, l'aceent étant porté sur le seul Attis. L'objection est de peu de poids, car, sur le vase qu'il ornait, le médaillon pourait être associé à un second, réservé à la léesser. L'existence de semblables médaillons est largement attestée, mème parmi ceux que l'on at tribue à Felix. L'un d'entre eux, d'un diamètre de $70 \mathrm{~mm}$, montre Cybèle assise sur un lion marchant vers la droite : il est connu en cinq exemplaires, dont un de Lyon et trois de Vienne $e^{29}$. En autre, plus tardif et plus grand, et d'un autre céramiste, montre la déesse dans la mème posture, les pieds posés sur un globe. Au-dessous, deux oiseaux encarlrent un objet ovoïde. Ce fragment a été trouvé dans les ruines d'une boutique mitoyenne du sanctuaire lyonnais de cybile ${ }^{30}$.

26 Ibid., p. $3: 2-37$.

27 Ibid., 1. $37-41$.

28 Ibial., p. 46-47.

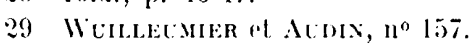

30 Ibid., no 305. 
9. - Dans cetle étude des médaillons mystiques, on ne négligera pas celui qui, portant la signature de Felix, ligure une scène demeurée longtemps énigmatique el sur laquelle une identifiration récente n'apporte pas une lumiere totale.

Un fragment de médaillon trouvé à Lyon figure un dieu marin. Neptune sans doute, étendu, appuyé sur une ancre. Derrière lui, sur un podium, s'érige un char tiré par quatre éléphants. et dont la caisse est ornée de petits personnages en cortège. Sur ce char, sont deux individus mutilés dont le second pourrait être une Victoire. Autour, apparaissent des structures archilecturales. Le relief de Lyon, très fruste, a été éclairé par la confrontation avec une épreuve trouvée à Cologne. Infiniment. plus nette, encore que plus mutilée que celle de Lyon, cette épreuve permet de lire, outre la signalure de Felix, les mots Portus Augusli qui expliquent les éléments de constructions portuaires servant de fond a la scine ${ }^{31}$.

Quelle signification pouvait avoir. aux yeux des Lyonnais à qui était destiné le médaillon. le port d'Auguste que l'on doit identifier à celui d'Ostie, construit par Claude et à qui Néron altribua le nom sous lequel il est connu? Il n'est pas interdit de supposer que la scène, dont certains éléments nous échappent, était en relation avec la légende de la Grande Mère qui, au Portus Augusti, avail posé pour la premiere fois le pied sur la terre romaine. Connaissant le goût de Felix pour les sujels relatifs aux cultes orientaux. peut-être expliquerait-on, par référence à l'arrivée de Cybèle en occident, le choix rlu sile métroaque d'Ostie.

Si les auteurs du présent article devaient justifier son utilité, ils pourraient au moins prétendre que, jusqu'ici, l'on n'avait guère songé à rechercher dans le répertoire limité des médaillons d'applique rhodaniens une source de documentation relative aux religions à mysteres pratiquées dans cette région. Or cette documentation est précieuse. Sur le culte d'Isis, notre information était des plus médiocres ret nous décourrons que les isiaques de Iyon et de: Vienne avaient accoutumé de trainer processionnellement le char de leur déesse, que préédait un myste portant le masque d'Anubis. Pour la religion de Cybèle, le bénéfice est moindre parce que l'on connaissait mieux l'importance prise par cette divinité dans les deux villes rhodaniennes. Toutefois, l'éclairage portait plus sur le personnage de la déesse-mère que sur celui de son fils. Le médaillon étudié redonne la place qu'il mérite au dieu sanglant qui meurt et renaît à l'équinoxe de printemps. Une telle information relative au culte phrygien en Occident ne pouvait survenir avec plus de bonheur qu'au moment où l'on envisage le dégagement exhaustif des vestiges de l'immense sanctuaire lyonnais de Cybèle ${ }^{32}$.

Amable Arons et Ilugues Verter.

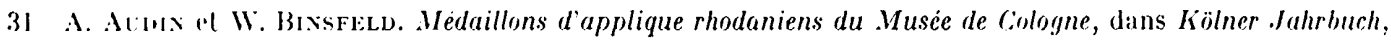
1964, p. 14-18 at pl. I. A. Alföl.DI, Die alexandrinischen Götter..., pl. 10, 2, 3.

32 A. Atrox, le sanctuaire lyonnais de Cybèle, dans Bulletin des musées el monuments lyonnais, $1965, \mathrm{n}^{\circ} 4$, p. 299-308. Ia ville de Jayon vient, an 1972, dacheter l'ensemble du terrain couvrant le sancluaire de Cybile. 